\title{
Structure of the analytical physical theories
}

\author{
Mário J. de Oliveira*1] \\ ${ }^{1}$ Universidade de São Paulo, Instituto de Física, Rua do Matão, 1371, 05508-090 São Paulo, SP, Brasil.
}

Received on April 26, 2021. Accepted on May 26, 2021.

\begin{abstract}
We present an analysis of the first analytical physical theories developed after the introduction of calculus. The underlying framework of these theories is the differential and integral calculus in the form developed by Leibniz. We point out the fundamental laws, or principles, or postulates upon which they are founded, and show some results or laws that are derived from them. We point out the main concepts of the theories drawing attention to the primitive or primary concepts, which are those undefinable. The theories that we describe are those that emerged just after the introduction of calculus, from Varignon to Navier. The areas of these theories include mechanics, electricity, magnetism, light, propagation of sound and propagation of heat.
\end{abstract}

Keywords: Physical theories, analytical theories, analytical mechanics.

\section{Introduction}

A scientific theory can be understood as a symbolic framework whose structure is based on a set of fundamental laws, or principles, or postulates, from which we derive other laws by deductive reasoning. The framework also contains the concepts of the theory, some of them being the primitive or primary concepts, which are undefinable. An essential feature of the theory is the list of correspondence between the symbolic abstractions of the theory and the real objects, which is usually called interpretation 1. Without the correspondence, a scientific theory is is just an abstraction that we call mathematics. In a previous paper [1, we have examined, in the light of this meaning of a scientific theory, the geometric physical theories from Euclid to Newton. Here we examine the analytical physical theories from the mechanics of Varignon, which appeared in the very beginning of the eighteenth century, until the fluid mechanics of Navier, in the first half of the nineteenth century [2-10.

The underlying mathematics of the physical theories up to the seventeenth century was mainly geometry [1. This was the case of the scientific theories developed by Galileo, Descartes, and Newton. When Galileo declared that mathematics was the language of nature, he meant geometry. However, there were important differences in the use of geometry by these authors. Descartes employed geometry in association with algebra, a method that came to be known as analytical geometry. Newton, in his Principles, used geometry in association with a limiting procedure, called the method of first and last ratios, to determine the tangents of curves and areas of geometric figures.

\footnotetext{
* Correspondence email address: oliveira@if.usp.br
}

In the last quarter of the seventeenth century, a powerful method emerged which replaced geometry as the underlying mathematics of physical theories [1]. The method called differential and integral calculus was independently invented by Newton and Leibniz. It consisted of a convenient procedure of dealing with evanescent quantities which allowed the determination of tangents of curves and areas of geometric figures. The evanescent quantities used by Leibniz were algebraic quantities that he called differentials. He introduced the notation $d x$ for the differential of a variable $x$, which is the notation that we use today. If $y$ and $x$ are the rectangular coordinates of a point in a certain curve, the tangent at this point is the ratio $d y / d x$. Newton called this ratio fluxion but he used a notation distinct from that of Leibniz. The method of first and last ratios used by Newton in the Principles may be understood as a geometric version of calculus.

Just after its invention, the infinitesimal calculus in the form given by Leibniz was applied by Leibniz himself and by Jacob and Johann Bernoulli to solve mechanical problems, an example of which was the brachistochrone problem [2]. It also served as the underlying mathematics for the physical theory that appeared from the beginning of the eighteenth century onward. We call these theories analytical theories as they use infinitesimal analysis which is another name for calculus. These include the theories related to the areas already treated by the geometric theories such as mechanics and optics, but include new areas such as sound, electricity, magnetism, and propagation of heat.

Our critical analysis is concerned mainly with theories. We point out their principles and primary concepts and present the derivation of some laws and concepts. The development of scientific ideas and theories is not free from discussions among scientists about their 
viewpoints. For instance, a debate appeared at the end of the seventeenth century about the appropriate way of measuring the mechanical effects, whether by the quantity of motion, which is mass multiplied by the velocity, or by the vis viva, which is the mass multiplied by the square of the velocity 9 . Some of these viewpoints will be also the object of our analysis. We will stick as closely as possible to the original terminology and the way the differentials were handled. We use the same mathematical symbols with the exception of the use of the slash symbol which, in some cases, will replace the usual horizontal fraction sign.

\section{Varignon}

Pierre Varignon was born in 1654 at Caen, France. He studied at the Jesuit College in Caen and at the University of Caen, where he earned a degree in 1682. He became a priest in 1683 and left Caen for Paris in 1686. The success of his first book, on the subject of statics, published in 1687 lead him to be appointed professor of mathematics at the Collège de Quatre-Nations in Paris in 1688. He lived in Paris until his death in 1722 [11. His main contribution was in the area of statics where he emphasized the principle of the composition of forces by the rule of the parallelogram [11.

The first works of Varignon did not involve the infinitesimal calculus. However, by 1700 he already had enough knowledge of infinitesimal calculus to write a series of three papers on mechanics where he applied the new mathematical tool in the form developed by Leibniz [9]. These papers [12 14, published in 1700, were based on the mechanics of Newton's Principles and it is possible that they are the first papers where the Newton laws of motion were written in the form of the infinitesimal calculus developed by Leibniz 9 .

The first paper [12] deals with the motion of a body along a straight line under the action of a force. Varignon starts by introducing two rules concerning the motion of a body along a straight line. The first rule is

$$
v=\frac{d x}{d t}
$$

and relates the velocity $v$ with the element of space $d x$ and the element of time $d t$. The increment of the velocity is $d v=d^{2} x / d t$ where $d t$ is constant, that is, $t$ is considered to be the independent variable. The second rule is,

$$
f=\frac{d v}{d t}
$$

or $f=d^{2} x / d t^{2}$, and relates the force $f$ with the increase in velocity. The elimination of $d t$ between the two rules written as $d x=v d t$ and $f d t=d v$ gives $f d x=v d v$.

The first rule is understood as the definition of velocity. The second rule is Newton's law for rectilinear motion with $f$ understood as the force divided by the

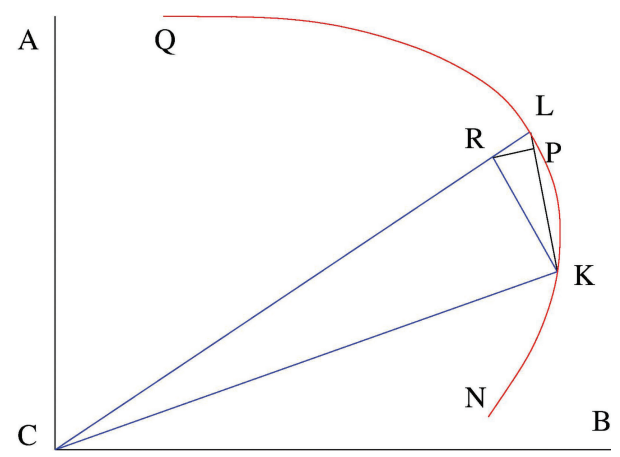

Figure 1: QLN is the trajectory of a body acted by a central force towards the center $C$. The point $K$ is close to the point $\mathrm{L}$. The distance $\mathrm{CR}$ and $\mathrm{CK}$ are equal and RP is perpendicular to $\mathrm{LK}$.

mass of the body. Varignon remarks that the two rules (1) and (2) give at once the proposition 39 of book 1 of Newton's Principles. The integration of equation $f d x=v d v$ gives

$$
\int f d x=\frac{1}{2} v^{2}
$$

or

$$
v=\sqrt{2 \int f d x}
$$

which is the first part of proposition 39. Replacing this result in $d t=d x / v$, and after integration,

$$
t=\int \frac{d x}{\sqrt{2 \int f d x}},
$$

which is the second part of proposition 39

The second paper [13] deals with the motion of a body along a curved line under the action of a central force. Let QLN be the trajectory of a body, L its position at a certain instant of time, and $\mathrm{K}$ its position after an elementary interval of time $d t$, as shown in Figure 1 The distance LK is $d s$ and the velocity of the body is

$$
v=\frac{d s}{d t}
$$

from which follows that $d v=d^{2} s / d t$, considering $d t$ constant. The distance of the point $\mathrm{L}$ to the center of force $\mathrm{C}$ is denoted by $r$ and $\mathrm{LR}$ is $d r$. Considering that the triangles LRK and RPL can be taken as similar, we may write $\mathrm{LP} / \mathrm{LR}=\mathrm{LR} / \mathrm{LK}$ or $\mathrm{LP}=d r^{2} / d s$. Denoting by $f$ the force towards the center $\mathrm{C}$, its component along LK will be $f(d r / d s)$. The increment $d v$ in the velocity will be proportional to the force along LK and to the increment in time $d t$ so that $d v=f(d r / d s) d t$ from which we get

$$
f=\frac{d s}{d r} \frac{d v}{d t}
$$


If we recall that $v=d s / d t$ and that $d v=d^{2} s / d t$, then the equation (7) can be written in the equivalent forms

$$
f=\frac{d s}{d r} \frac{d^{2} s}{d t^{2}}=v \frac{d v}{d r} .
$$

Varignon calls (6) and (7) the first and second rules of the motion along a curved line. The first rule is the definition of velocity. The second rule is the Newton's law of motion related to the tangential component of the force. Varignon uses the second rule to determine the force that acts on a body that follows a given curve, assuming that it is central. To this end he uses, in addition, another relation valid for central forces that was demonstrated by Newton. This relation states that the line connecting the body to the center of force sweeps equal areas in equal time. Denoting the length of the line segment RK by $d z$, then the area swept in an interval of time $d t$ is half $r d z$, considering that RK is perpendicular to CL. In analytical form, the relation is thus $r d z=\gamma d t$ where $\gamma$ is a constant which Varignon sets equal to unity.

The first example of a curved trajectory is an ellipse where the force is directed towards the center of the ellipse. The differential equation of an ellipse of major axis $a$ and minor axis $b$ is written in terms of the variables $z$ and $r$ as

$$
d z=\frac{a b d r}{\sqrt{\left.\left(a^{2}-r^{2}\right)\left(r^{2}-b^{2}\right)\right)}} .
$$

Here $r$ is the distance to the center of the ellipse. From this relation we determine $d s=\sqrt{d r^{2}+d z^{2}}$, which is

$$
d s=\frac{r d z}{a b} \sqrt{a^{2}-b^{2}-r^{2}} .
$$

Taking into account that $r d z=\gamma d t$ and that $v=d s / d t$, we obtain

$$
v=\frac{\gamma}{a b} \sqrt{a^{2}-b^{2}-r^{2}} .
$$

Now we use $f=v d v / d r$ to find

$$
f=-\frac{\gamma^{2}}{a^{2} b^{2}} r,
$$

that is, the force is proportional to $r$, the distance of the body to the center of force.

In the third paper [14, Varignon treats the problem of finding the force acting on a body following an ellipse but now the force is directed towards one focus of the ellipse. The differential equation that describes an ellipse of major axis $a$ and minor axis $b$ is

$$
d z=\frac{b d r}{\sqrt{2 a r-r^{2}-b^{2}}},
$$

where here $r$ is the distance to the focus. From this relation we determine $d s=\sqrt{d r^{2}+d z^{2}}$ which is

$$
d s=\frac{d z}{b} \sqrt{2 a r-r^{2}} .
$$

Taking into account that $r d z=\gamma d t$ and that $v=d s / d t$, we find

$$
v=\frac{\gamma}{b r} \sqrt{2 a r-r^{2}} .
$$

Now we use $f=v d v / d r$ to reach the result

$$
f=-\frac{a \gamma^{2}}{b^{2} r^{2}},
$$

that is, the force is proportional to the inverse of distance squared.

Varignon remarks that the inverse square law given by (16) is in accordance with the propositions 11,12 and 13 of book 1 of Newton's Principles. These propositions, demonstrated by Newton from his laws of motion, states that the bodies following an ellipse, a hyperbole, and a parabola with forces directed to their focus follow the inverse square law. Varignon remarks also that it is in accordance with the results given by Leibniz in a paper published in 1689, two years after the Principles. Based on differential geometry Leibniz showed in this paper the inverse square law for an ellipse 15 .

\section{Daniel Bernoulli}

Daniel Bernoulli was born in 1700 at Groningen, Netherlands, to where his family had moved some years earlier coming from Basel, Switzerland. At the age of five his family returned to Basel. He was taught mathematics by his father and by his older brother. He studied medicine at Basel, Heidelberg, and Strasbourg and obtained his doctorate in 1721 at the University of Basel. He went to St. Petersburg in 1725 as a professor of mathematics where he stayed for more than seven years. In 1733 he returned to Basel and thereafter he lived permanently in this city, where he died in 1782 [16. He lectured in botany and physiology at the University of Basel, and from 1750 he held the chair of physics in this university [16. His chief work is Hydrodynamics, published in 1738, but he published on medicine, mathematics, mechanics and theory of probability.

In his Hydrodynamics 17-19, Bernoulli presents a theory of motion of incompressible fluids, introducing the concept of pressure for fluids in motion, or hydrodynamic pressure. The novelty from previous works on the subject is the consideration of both pressure and motion [20. He treated basically the flow of fluids through tubes and the fluid outflow through orifices. His theory of fluid dynamics is based on the principle of the conservation of the living force which he stated more precisely as an equality between the actual descent and the potential ascent. In modern terms, this is understood as the conservation of the sum of kinetic energy and potential energy. He went on to say that this principle was formulated by Huygens, which in turn was a generalization of a law established by Galileo for the motion of bodies in inclined planes. 


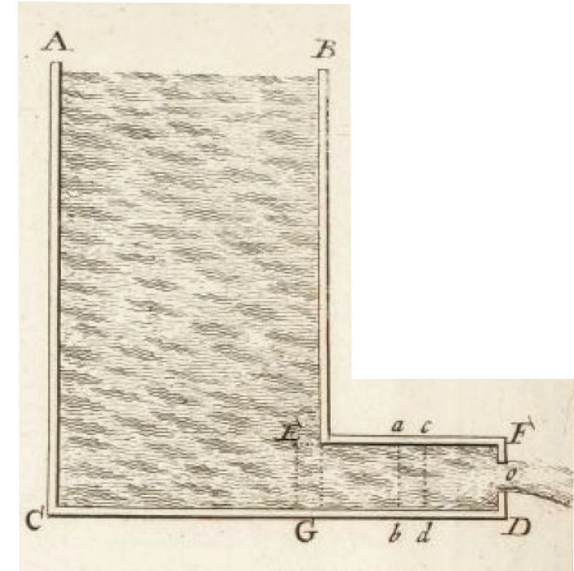

Figure 2: An illustration of Hydrodynamics [17] used in the demonstration of the Bernoulli equation. The vessel $A B G C$ is kept full of water, and $o$ is the hole through which the water escapes at constant velocity.

The statement given by Bernoulli of the principle formulated by Huygens is as follows. If a certain number of bodies begin to move from rest, the velocity acquired by each one is such that the sum of the products of their masses by the square of their velocities is proportional to the vertical height through which their center of gravity descends, multiplied by the total mass.

Bernoulli assumes another principle which, according to his words, he conceived by thought. If the fluid is divided into layers perpendicular to the direction of movement then the particles of the same layer have the same velocity from which follows that the speed of the fluid is inversely proportional to the area of the layer. This principles should be understood as the conservation of mass.

The hydrodynamics formula known as Bernoulli equation is derived in paragraph 5 of section 12 of the Hydrodynamics. He considers a very large vessel which is kept full of water, having a horizontal cylindrical tube, as shown in Figure 2. At the end of the cylindrical tube there is an orifice through which the water escapes at a constant velocity. It is required to find the pressure on the walls.

Bernoulli points out that the obstacle created by the smallness of the orifice causes an over-pressure on the tube, which is transmitted to the walls. He then assumes that the over-pressure is proportional to the acceleration, or to the increase in velocity, that would occur if the obstacle is removed. The acceleration is determined as follows 3, 10].

Let $v$ be the velocity of the fluid in the tube Ed so that the interval of time for a particle to traverse ac is $d t=d x / v$ where $d x$ is the length ac. The volume of the region acdb is $A d x$ where $A$ is the area of the cross section, and the corresponding mass is $\rho A d x$, where $\rho$ is the density of the fluid. The increase of living force during the interval of time $d t$ has two parts. One part comes from the portion of fluid entering the tube through EG, which is equal the mass of acdb multiplied by the velocity squared, or $(\rho A d x) v^{2}$. The other part comes from that part of the fluid leaving the tube Ed, which is $(\rho A \ell)\left[(v+d v)^{2}-v^{2}\right]$ or $2 \rho A \ell v d v$ where $\ell$ is the distance Ea. The total increase of living force, which is the actual descent, is thus $\rho A\left(d x v^{2}+2 \ell v d v\right)$. The corresponding potential ascent is proportional to the mass $\rho A d x$ multiplied by the height $h$ of the surface $\mathrm{AB}$ above the axis of the tube, which we write as $2 g(\rho A d x) h$, where $2 g$ is a constant of proportionality. But this quantity should be equal to the actual ascent at the hole which is the mass times the square of the velocity $v_{0}$ at the hole, or $(\rho A d x) v_{0}^{2}$.

By the principle of living force, the actual descent equals the potential ascent and

$$
d x v^{2}+2 \ell v d v=d x v_{0}^{2}
$$

or

$$
v \frac{d v}{d x}=\frac{v_{0}^{2}-v^{2}}{2 \ell} .
$$

We now observe that the left hand side of this equation is the acceleration $d v / d t$ because as $d t=d x / v$ it follows that $d v / d t=v d v / d x$. Therefore, the over-pressure is proportional to the right hand side of equation (18). To determine the coefficient of proportionality, Bernoulli argues that when the orifice is infinitely small the velocity $v$ is negligible compared to $v_{0}$ and the over-pressure becomes the hydrostatic $\rho g h=\rho v_{0}^{2} / 2$. Therefore, the over-pressure, which is the difference of the pressure $p$ in the tube and the pressure $p_{0}$ in the hole, will be

$$
p-p_{0}=\frac{1}{2} \rho v_{0}^{2}-\frac{1}{2} \rho v^{2},
$$

which is the Bernoulli equation.

The right hand of equation 19 can yet be written as $\rho g h\left(n^{2}-1\right) / n^{2}$ where $n$ is the ratio between the area of the cross section of the tube and the area of the orifice. Bernoulli did not present his result in the modern form given by equation 19 but saying that the pressure is proportional to $h\left(n^{2}-1\right) / n^{2}$.

\section{Euler}

Leonhard Euler was born in 1707 at Basel, Switzerland. At the age of thirteen he entered the University of Basel where he received a master degree in 1723 and a doctoral degree in 1726 . He accepted a position in St. Petersburg where he arrived in 1727, becoming a professor of physics in 1731. He was offered a post in Berlin to where he moved in 1741, living there for 25 years. In 1766, he accepted an invitation to return to St. Petersburg where he spent the rest of his life. He died there in 1783 [21]. Euler wrote a vast number of papers in various branches of mathematics and physical sciences, 
particularly in infinitesimal calculus, geometry, algebra, graph theory, mechanics, fluid statics, fluid dynamics, optics, and astronomy. He is most remembered as a leading mathematician of the eighteenth century but his contribution to physics is as important [22].

\subsection{Dynamics of a particle}

In 1736, Euler published his Mechanics [23, 24], a treatise in two volumes on the dynamics of a particle under the action of a force. The first volume concerns the unconstrained motion, or free motion, and the second, the constrained motion. In the preface of the treatise, Euler makes it clear that he uses the analytical method. By analytical method, he meant the infinitesimal analysis, understood as the differential and integral calculus in the form advanced by Leibniz.

According to the fundamental law of motion employed by Euler, a force acting on a particle changes the motion of that particle. By a change of motion it is meant not only the change in the speed but also in the direction of motion. Thus in the absence of force the body remains at rest or moving with a constant speed along a certain direction. In a more modern terminology we would say that the force changes the velocity, understood as vector quantity, which comprises its magnitude, or speed, and its direction. The composition of motion follows the rules of parallelogram, adopted by Euler, which in the modern terminology corresponds to the sum of vector velocities. For the sake of brevity and clearness, we will use the term velocity in the place of motion whenever it is pertinent.

\section{Tangential and normal forces}

Euler analyzes initially the change of motion along a straight line in which case only the speed changes. If the particle moves is a straight line then, in accordance with the law of motion, the increase $d v$ in the speed during an element of time $d t$ is

$$
d v=f d t
$$

where $f$ is the force divided by the mass of the particle. Euler remarks that this relation was considered by Daniel Bernoulli, who wrote this equation in a paper about the composition of forces published in 1728 [25]. Taking into account that the elementary distance $d y$ is related to the velocity by $d y=v d t$, then $v d v=f d y$, which relates the speed and distance. From the relation $d y=v d t$ we may determine the differential of the second order $d^{2} y=d v d t$, considering that $t$ is the independent variable, or in the language used by Euler, that $d t$ is constant. Replacing $d v=f d t$ in this equation we find

$$
d^{2} y=f d t^{2}
$$

which is an expression of the law of motion equivalent to $d v=f d t$.

If $f$ is constant, as in a free fall, the integration of $v d v=f d y$ gives $v^{2}=2 f y$, if the particle is at rest

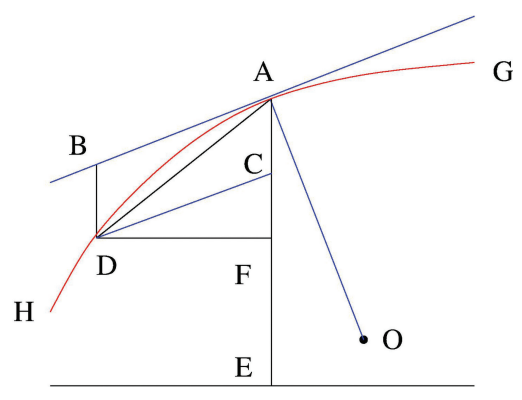

Figure 3: A particle following the trajectory $G A H$ is acted at $A$ by a force in the direction $A E$. The line segment $B A$ is tangent to the trajectory at $A$, and $A O$ is perpendicular to $B A$. The point $\mathrm{O}$ is the center of the osculating circle.

when $y=0$. The time the particle takes to travel the distance $y$, Euler obtains it by integrating $d t=d y / v$ where $v=\sqrt{2 f y}$. The result is $t=\sqrt{2 y / f}$, or $y=f t^{2} / 2$, which is equivalent to $y=v t / 2$.

We treat now the change of speed of a particle when it moves along a non rectilinear trajectory under a force that is not in the direction of the motion, as shown in Figure 3. A and D are two successive positions of the particle differing in time by an element $d t$. The velocity of the particle at the point $\mathrm{A}$ has the direction $\mathrm{AB}$, tangent to the curve, and $\mathrm{AB}$ equals $v d t$, where $v$ is the speed at $\mathrm{A}$. In the absence of force, the particle would be found at the point $B$. As a result of the action of the force, the particle will be at the point $\mathrm{D}$ such that ABDC is a parallelogram. The side AC of the parallelogram has the direction of the force and its length is the distance traveled by the particle if the particle were at rest at the point $\mathrm{A}$, and is equal to $f d t^{2} / 2$, where $f$ is the magnitude of the force divided by the mass.

When the particle reaches the point $\mathrm{D}$, its speed will be $v+d v$ where $d v$ is the increase in the speed from $\mathrm{A}$ to $\mathrm{D}$. Considering that the angle BAD is small, we may write the distance $\mathrm{AD}$ as given by $(v+d v / 2) d t$, so that $\mathrm{AD}-\mathrm{AB}=d v d t / 2$. For the same reason, the difference $\mathrm{AD}-\mathrm{AB}$ can be set equal to $\mathrm{BD} \cos \alpha$ where $\alpha$ is the angle $\mathrm{BAC}$ and we reach the result $d v=2(\mathrm{BD} / d t) \cos \alpha$ or

$$
d v=(f \cos \alpha) d t
$$

because $\mathrm{BD}$ equals $\mathrm{AC}$ and $\mathrm{AC}=f d t^{2} / 2$. As $\alpha$ is the angle between the velocity and the force, the result $(22)$ says that the change in speed is related to the component of the force in the direction of the velocity.

Let $\mathrm{AO}$ be perpendicular to $\mathrm{AB}$ and $\mathrm{O}$ be the center of a circumference passing through the points $\mathrm{A}$ and D. When D approaches A, the circumference becomes the osculating circumference. Considering that the angle $\mathrm{AOD}$ is twice the angle $\mathrm{BAD}$, the following geometric relation is valid: $\mathrm{DA} / 2 \mathrm{AO}=(\mathrm{AC} \sin \alpha) / \mathrm{DA}$. This relation is equivalent to $(\mathrm{DA})^{2} / R=f d t^{2} \sin \alpha$ where $R$ is the radius $\mathrm{AO}$ of the osculating circumference, and we 
recall that $\mathrm{AC}=f d t^{2} / 2$. When $\mathrm{D}$ approaches $\mathrm{A}, \mathrm{AD} / d t$ becomes $v$ and we get

$$
f \sin \alpha=\frac{v^{2}}{R} .
$$

If the particle follows a circumference of radius $R$ with constant speed $v$, this equation gives the formula for the centripetal force $f=v^{2} / R$ because in this case $\alpha$ is a right angle.

Denoting by $y$ the distance from EA, then $d^{2} y=$ $-f d t^{2}$ and equation 22 becomes $d^{2} y \sin \alpha=-d s^{2} / R$ where we used $d s=v d t$. The differential $d s$ is identified with DA and equals $\sqrt{d x^{2}+d y^{2}}$ where $d x=\mathrm{DF}$ and $d y=\mathrm{AF}$. From these relations it follows that $\sin \alpha=$ $d x / d s$ and we get $d^{2} y(d x / d s)=-d s^{2} / R$, which leads to the formula

$$
R=-\frac{d s^{3}}{d x d^{2} y}
$$

for the radius of the osculating circumference or radius of curvature.

\section{Central forces}

In chapter five of the first volume of the Mechanics, Euler analyzes the motion of a particle under a central force. A particle moves along a trajectory FAG under a central force toward $\mathrm{C}$ as shown in Figure 4 . At a certain instant of time the particle is at the position $\mathrm{A}$ with speed $v$. The line DAB is tangent to the trajectory and the length of $\mathrm{AB}$ is $v d t$ where $d t$ is a small interval of time. The following notations are used: $\mathrm{AE}=d z, \mathrm{~EB}=d r$, $\mathrm{AB}=d s, \mathrm{AC}=r$, and $\mathrm{CD}=p$. Considering that the angle $\mathrm{ACE}$ is small, the triangle AEB can be taken similar to CDA from which follows $p d s=r d z$. Notice that by denoting the elementary angle ACB by $d \theta$ then $d z=r d \theta$, and $r$ and $\theta$ are understood as polar coordinates.

The derivation that we are about to carry out uses the relation $R=r d r / d p$ involving the osculating radius $\mathrm{OA}=R$. Denoting by $\alpha$ de angle $\mathrm{CAD}$, then $p=R \sin \alpha$ from which we get $d p=d r \sin \alpha$ or $d p=d r(d z / d s)$. As $\mathrm{B}$ approaches $\mathrm{A}$, the angle $\mathrm{ACB}$ can be considered to be equal to the angle AOB from which we obtain the relation $\mathrm{AE} / \mathrm{AC}=\mathrm{AB} / \mathrm{AO}$ or $d z / r=d s / R$. From these results we get the desired relation $d p=d r(r / R)$.

The change in speed $d v$ is related to the tangent component of the force, as given by equation 22 . This result allows us to write $d v=-f(d r / d s) d t=$ $-f d r / v$, or

$$
v d v=-f d r,
$$

where $f$ is the force divided by the mass and the minus sign indicates that the force is toward $\mathrm{C}$, in opposition to the increase in $r$. If we let $u$ be the integral of $f d r$, the integral of equation 25 gives

$$
\frac{v^{2}}{2}=-u+b
$$

where $b$ is a constant.

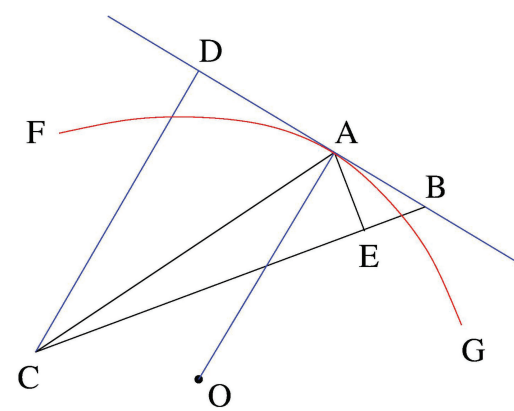

Figure 4: A particle following the trajectory FAG is acted at $A$ by a force in the direction $A C$. The line segment DAB is tangent to the trajectory at $A$ and $A O$ is perpendicular to $D A B$. The point $\mathrm{O}$ is the center of the osculating circle.

Another equation comes from the relation between the perpendicular component of the force and $v^{2} / R$, as given by equation 23, that is, $v^{2} / R=f(d z / d s)$. Using equation (25) to eliminate $f$ we found $d v / v=$ $-(d r / R)(d s / d z)$. Taking into account that $d s / d z=r / p$ and that $R=r d r / d p$ we find $d v / v=-d p / p$, which integrated gives $v p=k$ where $k$ is a constant. Using the relations $p d s=r d z$ and $d s=v d t$, we conclude that $r d z=k d t$ or $r^{2} d \theta=k d t$. But $r^{2} d \theta$ is twice the area of the elementary triangle $\mathrm{CAB}$, and $r^{2} d \theta=k d t$ describes the Kepler law of areas.

The equation that describes the trajectory is obtained by replacing $v=k / p$ into equation (26), which gives $k^{2} / p^{2}=-u+b$. Substituting $p=r d z / d s=r^{2} d \theta / d s$ in this equation, the result is

$$
\frac{k^{2} d s^{2}}{2 r^{4} d \theta^{2}}=-u+b
$$

As $d s^{2}=d r^{2}+r^{2} d \theta^{2}$ and $u$ is a function of $r$, then 27 is a differential equation involving $r$ and $\theta$. It can also be written in the equivalent form

$$
d \theta=\frac{k d r}{r \sqrt{2 r^{2}(b-u)-k^{2}}},
$$

the integration of which gives $\theta$ in terms of $r$. The time is obtained by the relation $r^{2} d \theta=k d t$, that is,

$$
d t=\frac{r d r}{\sqrt{2 r^{2}(b-u)-k^{2}}} .
$$

Euler considers several types of central forces. When the force is proportional to the distance, $f=c r, u=$ $c r^{2} / 2$, and

$$
d \theta=\frac{k d r}{r \sqrt{2 b r^{2}-c r^{4}-k^{2}}} .
$$

Defining the new variable $q=1 / r^{2}-b / k^{2}$, we have

$$
d \theta=-\frac{d q}{2 \sqrt{a^{2}-q^{2}}},
$$


where we are using the abbreviation $a^{2}=b^{2} / k^{4}-c / k^{2}$ The integration of this equation gives $q=a \cos 2 \theta$ and we reach the result

$$
a \cos 2 \theta=\frac{1}{r^{2}}-\frac{b}{k^{2}}
$$

Introducing the rectangular coordinates $x=r \cos \theta$ and $y=r \sin \theta$, this equation becomes

$$
a\left(x^{2}-y^{2}\right)+\frac{b}{k^{2}}\left(x^{2}+y^{2}\right)=1 .
$$

which describes an ellipse. The center of force is located at the point where $x$ and $y$ vanish. But this point is the center of the ellipse and we conclude that the center of force is located at the center of the ellipse.

If the central force is inversely proportional to the square of the distance, $f=c / r^{2}, u=-c / r$, and the equation 28 becomes

$$
d \theta=\frac{k d r}{r \sqrt{2 b r^{2}+2 c r-k^{2}}} .
$$

Defining the new variable $q=-1 / r+c / k^{2}$, we find

$$
d \theta=\frac{d q}{\sqrt{a^{2}-q^{2}}},
$$

where we are using the abbreviation $a^{2}=2 b / k^{2}+c^{2} / k^{4}$. The integration of this equation gives $q=a \sin \theta$ and we reach the result

$$
a \sin \theta=-\frac{1}{r}+\frac{c}{k^{2}}
$$

Using the rectangular coordinates, we get

$$
(1+a y)^{2}=\frac{c^{2}}{k^{4}}\left(x^{2}+y^{2}\right),
$$

which describes an ellipse. The center of force is located at the point where $x$ and $y$ vanish, but this point is not the center of the ellipse. It is a focus of the ellipse, as can easily be shown. Thus, in this case the center of force is located at one focus of the ellipse.

The constrained motion of a particle is treated in the second volume of the treatise. In addition to the forces determined by the constraint, the body may be acted by external forces. If the body is constrained to move along a straight line, not necessarily a straight line, as if there is no external forces, Euler shows that the body keeps the same speed. This results follows from the fact that the forces that results from the constraint is allways perpendicular to the trajectory. The absence of tangential force results by equation 22 in the constancy of the speed. In the proposition of this result, Euler excludes lines having adjointing elements with a finite angle, which would yield a nonzero tangential force.

In the following Euler discusses the constrained motion of a particle under an external forces. A particle is constrained to move along the trajectory $\mathrm{ADB}$ and

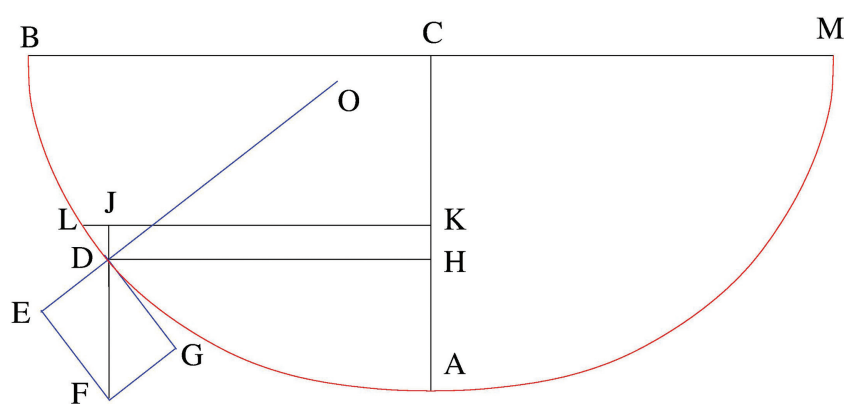

Figure 5: A particle following the trajectory BDAM is acted at $D$ by a force in the vertical direction DF. The line segment DG is tangent to the trajectory at $\mathrm{D}$ and $\mathrm{OD}$ is perpendicular to DG. The point $O$ is the center of the osculating circle.

is under the action of a vertical force along DF, as shown in Figure 5. We use the notations $\mathrm{AH}=y, \mathrm{HK}=d y$, $\mathrm{HD}=x, \mathrm{JL}=d x, \overrightarrow{\mathrm{DL}}=d s$. The force divided by the mass is $\mathrm{MF}=f$, its tangent component is $\mathrm{DG}=f(d y / d s)$ and its normal component is $\mathrm{DE}=f(d x / d s)$. We have seen above that the change in speed $d v$ is due to the tangential component of the force, $d v=-f(d y / d s) d t$, and as $d s=v d t$, one finds $v d v=-f d y$. Therefore, if the curve is given, that is, if $x$ is a function of $y$, we integrate the $f d y$ and the speed $v$ may be found along the trajectory. After $v$ has been obtained, we use the equation $d s=v d t$ to determine $t$,

$$
t=\int \frac{d s}{v} .
$$

Let us consider that the vertical force acting on the particle is the force of gravity. The force of gravity divided by the mass is denoted by $g$, which is constant. Then the integration of equation $c d c=-g d y$ gives $v^{2} / 2=-g(y-h)$ where $h$ is the value of height $y$ when the body has velocity zero. The time is determined by

$$
t=\int \frac{d s}{\sqrt{2 g(h-y)}} .
$$

If the trajectory BDAM of Figure 5 is a semi-circle, as happens to a simple pendulum, then $x=\sqrt{2 R y-y^{2}}$, $d x=(R-y) d y / x, d s=(R / x) d y$, and

$$
t=\int \frac{R d y}{\sqrt{2 g y(2 R-y)(h-y)}} .
$$

To find the time it takes for the body to descend from the rest to the lowest point of the trajectory, which is one quarter of the period of oscillation, the integration is performed from $y=h$ to $y=0$.

If $h$ is small,

$$
t=\frac{1}{2} \sqrt{\frac{R}{g}} \int \frac{d y}{\sqrt{y(h-y)}} .
$$

The integral is equal to $\pi$ from which follows the result $t=(\pi / 2) \sqrt{R / g}$. This is one quarter of the 
period of oscillation. Thus the period of small amplitude oscillations of a simple pendulum is $2 \pi \sqrt{R / g}$.

Euler determined the integral in 40 by an expansion in powers of $r=h / 2 R$. To this end he expands $1 / \sqrt{2 R-y}$ in powers of $y$ and then integrates each term in the expansion. The result for $4 t$, which is the period of oscillation, is

$$
2 \pi \sqrt{\frac{R}{g}}\left[1+\frac{r}{2^{2}}+\left(\frac{1.3}{2.4}\right)^{2} r^{2}+\left(\frac{1.3 .5}{2.4 .6}\right)^{2} r^{3}+\ldots\right] .
$$

Let us consider now that the trajectory BDAM of Figure 5 is a cycloid. The cycloid holds the property that its length from the lowest point A to a point of height $y$ is equal $\sqrt{8 a y}$. Therefore, the length of a part of the cycloid from a point of height $h$ to a point of height $y$ is $s=\sqrt{8 a h}-\sqrt{8 a y}$. From this result we get $d s=-\sqrt{2 a / y}$ which replaced in 39 gives

$$
t=\frac{\sqrt{a}}{\sqrt{g}} \int \frac{d y}{\sqrt{y(h-y)}},
$$

where the integration is performed from $y=h$ to $y=0$, given thus one quarter of the period of oscillation. This integral is the same as that found above and equals $\pi$ from which follows the result $t=\pi \sqrt{a / g}$. Thus the period of oscillation is $4 \pi \sqrt{a / g}$ and is independent of the amplitude of oscillation. Taking into account that the radius of curvature is $R=4 a$ the period of oscillation is $2 \pi \sqrt{R / g}$, which is the same as that of a simple pendulum of length $R$ executing small oscillations.

\subsection{Hydrodynamics}

Euler formulated an analytical theory of fluid mechanics in three successive papers published in 1757 [26 28. The first paper dealt with hydrostatics, and the second and third with hydrodynamics. The underlying mathematics is the differential and integral calculus, used in the analytical form developed by Leibniz. He also employed the notion of function that he had introduced before 29 . Euler formulates his theory for the static and dynamics of a fluid by an approach that differs from that used in his treatise on the dynamics of a particle. Instead of asking for the position and velocity of a particle at any instant of time, now a position in space is given and one asks for the velocity of the particle located at this point at a given instant of time. The latter form is known as Eulerian and the former as Lagrangian although both forms are due to Euler [30. Therefore, in accordance with the Eulerian form, the velocity and other properties such as the density of the fluid are considered to be functions of the position and time.

He starts by defining within the fluid a rectangular system of reference consisting of three orthogonal axes. The coordinates of a certain point of the fluid is denoted by $x, y$, and $z$. At each point of the fluid one associates the density $\rho$, the pressure $p$, and the three components $u, v$ and $w$ of the velocity, which are all considered to be functions of $x, y, z$, and $t$. In addition, the fluid may be acted by external forces such as gravity. The components of the forces per unit mass are denoted by $P, Q$, and $R$, an may depend on the coordinates $x, y$, and $z$.

Let us consider an element of volume $d x d y d z$ around a certain point. After an interval of time $d t$, the elements $d x, d y$, and $d z$ change to $d x+d u d t, d y+d v d t$, and $d z+$ $d w d t$, so that $d x d y d z$ changes to

$$
d x d y d z\left[1+d t\left(\frac{d u}{d x}\right)+d t\left(\frac{d v}{d y}\right)+d t\left(\frac{d w}{d z}\right)\right] .
$$

We remark that Euler encloses the ratio of differentials between parentheses to denote a partial differentiation. The density $\rho$ also changes to a new value which is determined recalling that the $x, y$, and $z$ increases by the amounts $u d t, v d t$, and $w d t$. The density changes to

$$
\rho+d t\left(\frac{d \rho}{d t}\right)+u d t\left(\frac{d \rho}{d x}\right)+v d t\left(\frac{d \rho}{d y}\right)+w d t\left(\frac{d \rho}{d z}\right)
$$

Considering that the mass in the new element of volume, given by the expression (44), is the mass in previous element of volume $d x d y d z$, then the product $\rho d x d y d z$ should be equal to the product of the expressions 44 and 45. from which follows

$$
\left(\frac{d \rho}{d t}\right)+\left(\frac{d \rho u}{d x}\right)+\left(\frac{d \rho v}{d y}\right)+\left(\frac{d \rho w}{d z}\right)=0 .
$$

This equation is referred to as the continuity equation because it reflects the continuity of the fluid which is another way to say that the mass is conserved.

If, as it may happen to some fluids, the new element of volume, given by the expression (44), is equal to the old one $d x d y d z$, then

$$
\left(\frac{d u}{d x}\right)+\left(\frac{d v}{d y}\right)+\left(\frac{d w}{d z}\right)=0
$$

Fluids obeying this equation are called incompressible. In modern terms the left hand side is the divergence of the velocity. Thus an incompressible fluid has zero divergence.

Next Euler seeks for the fluid equation, which involves the external forces and the pressure. According to the laws of motion, the force acting on a particle is proportional to the change of velocity. To find the change of velocity of a particle during an interval of time $d t$, one should take into account that in this interval the particle has move to a nearby point. Thus to find the change in velocity it is necessary to compare the velocity at the point $x, y$, and $z$, at time $t$ with that of the point $x+d x$, $y+d y$, and $z+d z$, at time $t+d t$. The increases in $u, v$, 
and $w$ during $d t$ are $X d t, Y d t$, and $Z d t$ where

$$
\begin{aligned}
X & =\left(\frac{d u}{d t}\right)+u\left(\frac{d u}{d x}\right)+v\left(\frac{d u}{d y}\right)+w\left(\frac{d u}{d z}\right), \\
Y & =\left(\frac{d v}{d t}\right)+u\left(\frac{d v}{d x}\right)+v\left(\frac{d v}{d y}\right)+w\left(\frac{d v}{d z}\right), \\
Z & =\left(\frac{d w}{d t}\right)+u\left(\frac{d w}{d x}\right)+v\left(\frac{d w}{d y}\right)+w\left(\frac{d w}{d z}\right) .
\end{aligned}
$$

Let us consider the parallelepiped of volume $d x d y d z$. The force due to the pressure $p$ along the direction $x$ is $p d y d z$ in one face and is $(p+(d p / d x) d x) d y d z$ in the opposite face so that the net force in the $x$ direction is $(d p / d x) d x d y d z$. Considering that the mass of the parallelepiped is $\rho d x d y d z$, the force due to pressure divided by the mass is $(1 / \rho)(d p / d x)$. To this term we have to add the component $P$ of the external force per unit mass along the $x$ direction. Thus the total force per unit mass along this direction will be $P-(1 / \rho)(d p / d x)$. Using the law of motion that the force divided by the mass equals the acceleration, one finds

$$
\begin{aligned}
& P-\frac{1}{\rho}\left(\frac{d p}{d x}\right)=X, \\
& Q-\frac{1}{\rho}\left(\frac{d p}{d y}\right)=Y, \\
& R-\frac{1}{\rho}\left(\frac{d p}{d z}\right)=Z .
\end{aligned}
$$

These equations are known as the Euler equations.

The three equations (51), 52, and (53) involving the forces, together with the continuity equation (46) and the equation that relates the pressure $p$ to the density $\rho$ comprise the five equations that encloses the theory of the fluid motion. Euler states that all discoveries in the field of hydrodynamics made by his predecessors, including Daniel Bernoulli, d'Alembert, and Clairaut, are consequences of his theory expressed in those four equations. Multiplying the equations (51), (52), and (53) respectively by $d x, d y$ and $d z$ and summing them we find

$$
P d x+Q d y+R d z-\frac{1}{\rho} d p=X d x+Y d y+Z d z,
$$

which encloses in one expression the three equations of the fluid motion.

The analysis of the equations of fluid motion is proceeded by considering that $d V=P d x+Q d y+R d z$ is an exact differential which means that $d V$ is the differential of a function $V$, called by Euler the effort of the soliciting forces. The equation (54) becomes

$$
d V-\frac{1}{\rho} d p=X d x+Y d y+Z d z
$$

He then considers that $u d x+v d y+w d z$ is an exact differential, or in modern terms, that the flow is irrotational.
This condition gives the relations $(d u / d y)=(d v / d x)$, $(d u / d z)=(d w / d x)$, and $(d v / d z)=(d w / d y)$. Replacing these results into the equations (48), (49), and (50), we find

$$
\begin{aligned}
& X=\left(\frac{d u}{d t}\right)+\frac{1}{2}\left(\frac{d \gamma^{2}}{d x}\right), \\
& Y=\left(\frac{d v}{d t}\right)+\frac{1}{2}\left(\frac{d \gamma^{2}}{d y}\right), \\
& Z=\left(\frac{d w}{d t}\right)+\frac{1}{2}\left(\frac{d \gamma^{2}}{d z}\right),
\end{aligned}
$$

where $\gamma^{2}=u^{2}+v^{2}+w^{2}$. Replacing these expressions into the equation (54), it becomes

$d V-\frac{1}{\rho} d p=\left(\frac{d u}{d t}\right) d x+\left(\frac{d v}{d t}\right) d y+\left(\frac{d w}{d t}\right) d z+\frac{1}{2} d \gamma^{2}$

From now we will depart from Euler and follow Lagrange in his treatise on Analytical Mechanics, published in 1788 [31], and introduce the velocity potential. As $u d x+v d y+w d z$ is an exact differential, Lagrange introduces the function $\phi$ of $x, y, z$, the velocity potential, such that $d \phi=u d x+v d y+w d z$. Therefore $u=(d \phi / d x), v=(d \phi / d y)$, and $w=(d \phi / d z)$, and the sum of the three terms on the right-hand side of the equation $\sqrt[55]{ }$ is the differential $d(d \phi / d t)$, and this equation simplifies to

$$
d V-\frac{1}{\rho} d p=d\left(\frac{d \phi}{d t}\right)+\frac{1}{2} d\left(\gamma^{2}\right),
$$

the integration of which gives

$$
V-\int \frac{1}{\rho} d p=\left(\frac{d \phi}{d t}\right)+\frac{1}{2} \gamma^{2}+C
$$

where $C$ is a constant of integration, which depends on $t$. This equation can be understood as the Lagrange integral of the Euler equation of motion (51), (52), and (53) for an irrotational fluid and conservative external forces.

In the steady flow, $(d \phi / d t)=0$, and $C$ becomes independent of $t$. If in addition, the density $\rho$ is constant, then

$$
V-\frac{p}{\rho}=\frac{1}{2} \gamma^{2}+C
$$

In modern terms $-V$ is the potential energy per unit mass and the equation 62 is the expression of the conservation of mechanical energy. If the external force is the force of gravity then $V=-g z$ where $z$ is the vertical distance from the ground

$$
\rho g z+p+\frac{1}{2} \rho \gamma^{2}=K
$$

which is known as the Bernoulli equation. 


\subsection{Theory of sound}

Sound has always been understood as associated to the motion of the intimate parts of the bodies particularly the air, which in the neighboring of the ear produces the sensation of hearing [32]. In 1660, Boyle conducted experiments with an air pump and observed the decrease in the intensity of sound as the air was being extracted from the vessel, and concluded that sound needs a medium for transmission, which in the experiments was the air 32 . Although one cannot properly derive this conclusion from the experiments, they became the standard explanation for the need of a medium for the propagation of sound 32 . The first attempt towards a theory of the propagation of sound appeared at the end of the seventeenth century. It was propounded by Newton 32 and appeared in section 8 of book 2 of his Mathematical Principles, published in 1687 [33 35].

The theory of Newton received further development from Lagrange and Euler. Here we will be concerned with the theory of sound as it was developed by these two physicists. We examine three papers of Lagrange 36 38 and three papers of Euler [39-41] on the subject of the propagation of sound, that were written around 1759 . The first paper of Lagrange stimulated Euler to develop his analytical theory on the propagation of sound [42]. In the first paper, Euler derived the one-dimensional wave equation which was then extended to two and three dimensions in the subsequent papers.

Lagrange opens the first paper stating that the nature and the laws of the propagation of sound is to be found in the study of the oscillations of intimate parts of elastic fluids, caused by disturbances imprinted on the sound bodies and communicated to the elastic surrounding media. The first theory based on these assumptions, according to Lagrange, was developed by Newton who derived a formula to determine the velocity of sound in air which he could compare with the experimental measurements. The main elementary question to solve in order to develop the theory, according to Lagrange, is as follows. Given several particles along a straight line that are in equilibrium in virtue of their mutual forces, determine their motion after they are disturbed.

This problem was in fact set out and solved by Newton in the section 6 of book 2 of the Principles [33 35]. He assumed that the elastic particles execute the same oscillatory motion and that the amplitudes and periods of oscillation are the same for all particles. The solution given by Newton allowed him to show that the velocity of sound $c=\sqrt{p / \rho}$, where $p$ is the pressure, which he called elastic force and $\rho$ is the air density. From this formula, Newton obtained a value of 979 feet per second for the velocity of sound in air. For comparison he mention the figures obtained from experiments which is 1142 feet per second.

Below we analyze the problem posed above and demonstrate the equation of motion advanced by Lagrange for the propagation of sound and reach the

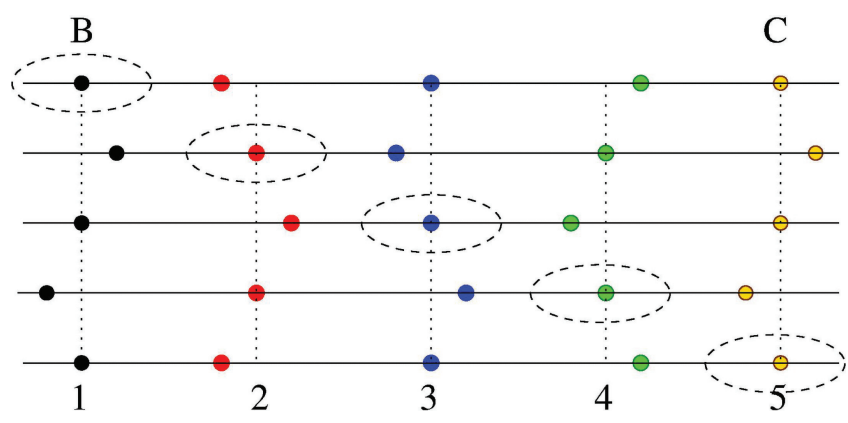

Figure 6: The particles oscillate along a straight line with the same period and the same amplitude. The arrangements of particles are shown at intervals of time equal to one fourth of the period of oscillations, from top to bottom. The vertical dotted lines indicate the central point of oscillation of each particle and they are equally spaced. The pulses are indicated by ellipses and correspond to regions where the particles are closer to each other.

Newton formula for the velocity of sound. The reasoning we use are similar to that of Lagrange which in turn is follows that of Newton. Figure 6 represents the oscillatory motion of a sequence of particles along a straight line that are constrained to move in this line. The central points of oscillations of the particles are distinct and are separated by the same distance $r$. The figure also shows the location of the pulses, understood as the region where the concentration of particle is the highest.

The forces acting on the particles are identified as that produced by the elasticity of air, and are in accordance with the Boyle law of gases that the pressure is inversely proportional to the volume. Let us consider a recipientin the form of a square box with length $\ell$ and area of the base equal to $A=r^{2}$, where $r$ is the side of the square base. If the length varies while the area remains unchanged, the force $f$ acting along its axis will be inversely proportional to $\ell$. Let us denote by $\rho$ the density of the air through which the sound propagates. In this case the force, denoted by $f_{0}$, is equal to $A$ times the atmospheric pressure $\rho g h$, where $h$ is the height of a homogeneous column of air and $g$ is the acceleration of gravity. When the density of the recipient is $\rho$, we assume that the length of the recipient is equal to $r$. Denoting by $m$ the mass of the air inside the recipient, we have then $\rho=m / r^{3}$, and considering that $A=r^{2}$, we find $f_{0}=m g h / r$. As $f / f_{0}=r / \ell$, we conclude that

$$
f=\frac{m g h}{\ell} .
$$

The force acting between two consecutive particles of mass $m$ separated by a distance $\ell$ is assumed by Newton and Lagrange to be that given by (64). As the deviation $y=\ell-r$ is considered to be small compared to $r$, the following expression is used

$$
f=m g h \frac{r-y}{r^{2}} .
$$


Let us denote by $y_{i}$ the position of the particle $i$ with respect to its central point of oscillation. The force that acts on this particle from left to right is that given by 65) with $y$ replaced by $y_{i}-y_{i-1}$, that is,

$$
\frac{m g h}{r^{2}}\left(r-y_{i}+y_{i-1}\right) \text {. }
$$

Similarly one finds the force that acts from right to left by replacing $y$ by $y_{i+1}-y_{i}$,

$$
\frac{m g h}{r^{2}}\left(r-y_{i+1}+y_{i}\right)
$$

The equation of motion for the particle $i$ is obtained by bearing in mind that the net force, the difference between the expressions (66) and (67), is the mass $m$ times the acceleration $d^{2} y_{i} / d t^{2}$, that is,

$$
\frac{d^{2} y_{i}}{d t^{2}}=\frac{g h}{r^{2}}\left(y_{i+1}-2 y_{i}-y_{i-1}\right),
$$

which is the equation found by Lagrange [36. In the place of $g$, Lagranges writes two times the height of a free fall divided by the square of the time of fall of a body.

To determine the velocity of the pulses we start by denoting by $\tau$ the time it takes for a pulse to travel a distance $r$. The velocity of the pulse, and thus the velocity of sound, will be $c=r / \tau$. The condition that determines $\tau$ is $y_{i+1}(t)=y_{i}(t+\tau)$ for any $i$. Replacing in 68, one finds

$$
\frac{d^{2} y_{i}}{d t^{2}}=\frac{g h}{r^{2}}\left[y_{i}(t+\tau)-2 y_{i}(t)-y_{i-1}(t-\tau)\right] .
$$

If $\tau$ is small, the terms between square brackets will equal the left hand side multiplied by $\tau^{2}$ which gives $g h \tau^{2} / r^{2}=1$ from which we find $c=r / \tau=\sqrt{g h}$. To reach the Newton formula, we recall that $p=\rho g h$ from which follows $c=\sqrt{p / \rho}$.

The continuous version of equation 68 was obtained by Euler in his first paper on the propagation of sound [39]. He sets $r=d x$ and writes

$$
\begin{gathered}
y_{i}-y_{i-1}=d x\left(\frac{d y}{d x}\right), \\
y_{i+1}-2 y_{i}+y_{i-1}=d x^{2}\left(\frac{d^{2} y}{d x^{2}}\right),
\end{gathered}
$$

which replaced in 68 gives the equation

$$
\left(\frac{d^{2} y}{d t^{2}}\right)=g h\left(\frac{d^{2} y}{d x^{2}}\right),
$$

which, says Euler, describes the propagation on sound along a thin tube. The variable $y$ is understood as a function of both $x$ and $t$, and Euler denotes a partial derivative by enclosing the ratio of differentials between parentheses. Let us write the equation 72 as

$$
\left(\frac{d^{2} y}{d t^{2}}\right)=c^{2}\left(\frac{d^{2} y}{d x^{2}}\right),
$$

where $c=\sqrt{g h}$. D'Alembert in his studied of the vibration of stretched strings had arrived in 1747 at this equation with $c=\sqrt{F / \mu}$ where $F$ is the force of tension and $\mu$ is the mass of the string per unit length [32, 43]. The derivation of d'Alembert was based on a calculation carried out by Taylor in 1713 [32, 44] concerning the force acting on a small segment of the string as a result of the force of tension and the curvature of the string.

A general solution of equation (73) is

$$
y=\Phi(x+c t)+\Psi(x-c t),
$$

where $\Phi$ and $\Psi$ are two arbitrary functions and was due to d'Alembert [43]. From this general solution, Euler drew the following results. If we set $t=0$, we get $\Phi(x)+\Psi(x)$ which represents the state of the air in the thin tube at the initial time. If we denote by $\Theta(x)$ the value of $y$ at $t=0$ and by $v(x)$ the velocity $(d y / d t)$ at $t=0$, then

$$
\begin{aligned}
\Phi(x)+\Psi(x) & =\Theta(x), \\
\Phi^{\prime}(x)-\Psi^{\prime}(x) & =\frac{v(x)}{c} .
\end{aligned}
$$

If we integrate the last equation we find

$$
\Phi(x)-\Psi(x)=\frac{\zeta(x)}{c}
$$

where $\zeta$ is the integral of $v$. The equations 75 and 777 determine $\Phi$ and $\Psi$ if the initial functions $\Theta$ and $\zeta$ are given.

To explain the propagation of sound in air, Euler consider a small perturbation of the air that occurs at $t=0$ in a small interval of the straight line along which the sound propagates. He argues that in this case $\zeta(x)$ vanishes and $\Theta(x)$ is zero except in the perturbation interval. The first condition gives $\Phi(x)=\Psi(x)=\Theta(x) / 2$ and

$$
y=\frac{1}{2} \Theta(x+c t)+\frac{1}{2} \Theta(x-c t) .
$$

Assuming that the perturbation occurs on an small interval around $x=0$ then $\Theta(x)$ vanishes except in that small interval. Using this result we see that $y$ vanishes except on the small intervals around $x=-c t$ and $x=c t$, which means that the perturbation, the sound, travels in both directions with velocity $c$. From the formula $c=\sqrt{g h}$, Euler determines the velocity of sound. The value of $g$ used by Euler is twice $15+5 / 8$ Rhine feet and $h=32 \times 800$ feet, which gives $c=894$ feet per second which is to be compared with the experimental value of 1100 feet per second. Faced with such a disagreement, already noted by Newton as we have seen above, Euler just remarks that no one has yet discovered the cause of the discrepancy.

In the second paper on the propagation of sound 40, Euler extends the wave equation (73) to two and three dimensions. In two dimensions, the equation describes 
the propagation of sound in the air between two plates separated by a small distance. The wave equation is expressed in terms of the variable $v$ which in modern terms is the divergence of the vector whose components are the displacement of a particle from its equilibrium position,

$$
\left(\frac{d^{2} v}{d t^{2}}\right)=c^{2}\left(\frac{d^{2} v}{d x^{2}}\right)+c^{2}\left(\frac{d^{2} v}{d y^{2}}\right)
$$

where $x$ and $y$ denotes the rectangular position of a point in the plane. In three dimensions, the wave equation reads

$$
\left(\frac{d^{2} v}{d t^{2}}\right)=c^{2}\left(\frac{d^{2} v}{d x^{2}}\right)+c^{2}\left(\frac{d^{2} v}{d y^{2}}\right)+c^{2}\left(\frac{d^{2} v}{d z^{2}}\right) .
$$

\section{Lagrange}

Joseph-Louis Lagrange was born in 1736 in Turin, Italy. His life is divided in three periods 45 . From his birth until 1766, he lived in Turin where from 1755 he held a position of professor at the Royal Artillery School. The second comprises the period from 1766 until 1787, when he worked at the Berlin Academy and wrote the remarkable and comprehensive treatise called Analytical Mechanics 31. In the third period, from the time he became a member of the French Academy of Sciences in 1787 until his death in 1813, he lived in Paris. In 1794 he became the first professor of analysis at the newly oppened École Polytechnique. He wrote in mechanics, sound,differential and integral calculus, and celestial mechanics.

\subsection{Analytical mechanics}

The Analytical Mechanics [31, published in 1788, is divided in statics and dynamics, and uses the differential and integral calculus in the analytical form introduce by Leibniz. Lagrange states at the beginning that the book has no geometric figures as the analytical method can be formulated without the need of geometry. In the following, we give a description of each part of the book.

\section{Statics}

The first part on statics is based on three principles. The first is the Archimedes lever rule. A straight and horizontal lever is in equilibrium if the weights are inversely proportional to theirs distances to the fulcrum. The second is the principle of composition of forces. Two forces of different directions acting on the same body are equivalent to a single force given by the rule of the parallelogram. The third is the principle of virtual velocities. Let us consider a system consisting of points in equilibrium which is acted by several forces. If a small movement is given to the system such that each point travels an infinitesimal distance that expresses its virtual velocity then the sum of the forces multiplied each one by the projection of the infinitesimal distance traveled by the point along the direction of the force is equal to zero. Each term of the sum is positive if the distance is traveled in the direction of the force and negative otherwise. Lagrange regarded this principle as an axiom of the theory and credited it to Johann Bernoulli on account of a letter sent by the latter to Varignon in 1717 reporting the principle.

Let us consider a system under the action of several forces $P, Q, R, \ldots$ and let us draw to each point where a force is applied a straight line parallel to the force. We denote by $d p, d q, d r, \ldots$ the projection along the these lines of the infinitesimal increments that result from a small perturbation of the system. The analytical form of the principle of virtual velocities is

$$
P d p+Q d q+R d r+\ldots=0 .
$$

In general, the displacement of the points where the forces are applied are not free from one another but have connections that lead to one ore more constraints between the variables $p, q, r$. That is, the differentials $d p, d q, d r$ are not independent of each other in general.

From the principle of virtual velocities (81), Lagrange derives two conditions for equilibrium. One is related to the motion of translation. If a free system is in equilibrium then the sum of the forces along each one of the three orthogonal axes vanishes. The other is relative to the rotational motion and is stated as follows. Consider an axis and a plane perpendicular to this axis. The moment of a force with respect to the axis is the product of the component of the force parallel to the plane with the distance to the axis. The moment of a force that would cause the rotation in a certain direction and that that would cause the rotation in the opposite direction are taken to be of the opposite signs. If the system is in equilibrium the sum of all the moments will vanish. We notice that the Archimedes lever rule stated above is in fact a particular case of this condition and in this sense it is not an independent principle but a derived law.

Let us consider that the left hand side of (81) is an exact differential of a function $\phi$, that is,

$$
d \phi=P d p+Q d q+R d r+\ldots
$$

The condition for equilibrium becomes $d \phi=0$ which means that the equilibrium occurs when the function $\phi$ is generically speaking a minimum or a maximum. According to Lagrange this constitutes the principle proposed by Maupertuis in 1740, called the law of rest of bodies.

To determine the state of equilibrium, Lagrange introduces the method of multipliers. Let $x, y, z, \ldots$ be the variables describing the rectangular coordinates of the points where the forces are applied. Let $L, M$, and $N$ be functions of these variables and let $L=0$, $M=0, N=0$ be the relation among these variables that describe the restrictions imposed on the system, valid also when it is perturbed. By differentiation, the restriction is equivalently written as $d L=0, d M=0$, 
and $d N=0$. Each one of these differentials is multiplied by an undermined quantity and added to equation (81). The result is

$$
P d p+Q d q+R d r+\lambda d L+\mu d M+\nu d N+\ldots=0 .
$$

which becomes the general equation of equilibrium. As the differentials in this equations are linear in the differentials $d x, d y, d z, \ldots$ the whole left hand side of this equation will also be linear in these differentials, that is, equation 83 becomes

$$
X d x+Y d y+Z d z+\ldots=0
$$

The introduction of the multipliers $\lambda, \mu$, and $\nu$ make the differentials $d x, d y, d z$ to be independent of each other and we conclude that $X, Y$, and $Z$ vanish. The equations $X=0, Y=0$, and $Z=0$ are called particular equations of equilibrium, and together with $L=0, M=0$, and $N=0$, they will provide $x, y, z$ at equilibrium as well as the values of $\lambda, \mu$, and $\nu$.

\section{Dynamics}

Lagrange opens the second part of the treatise by stating that the science of dynamics is due to modern investigations, particularly to Galileo who developed the basic principles, to Huygens who completed most of Galileo work and added his own contribution, and mainly to Newton who turned the discipline into a new science. He then added that the infinitesimal calculus enabled the investigators to reduce the laws of motion to analytical expressions and that the relation between force and motion became the main object of their work.

According to Lagrange, the first great study of mechanics where the analytical method was applied was Euler's Mechanics 23, published in 1736. Euler based the study of the dynamics of a particle on the formulas for the tangent and normal forces. In 1744, Euler published a book on the method of determining curves fulfilling minimum of maximum properties such as the isoperimetrical problem [46]. The variational method called the attention of Lagrange who gave his own contribution to the subject [47, 48] and envisaged its use as an approach to mechanics in connection with the Maupertuis principle of least action [49, 50. However, Lagrange did not formulated his mechanics based on this principle but used instead the d'Alembert principle.

From the d'Alembert principle which Lagrange adopted as the fundamental principle of his Analytical Mechanics, he obtains the following principles, which are in fact theorems or derived laws.

1. Conservation of living forces.

2. Conservation of the motion of the center of gravity.

3. Conservation of the motion of rotation,

or principle of areas.

4. The principle of the least quantity of action.
In modern terms the first three are know as the conservation of mechanical energy, the conservation of the linear momentum, the conservation of the angular momentum, respectively. The fourth principle was proposed by Maupertuis and is connected with an integral of the vis viva in time but the formulation of the principle as used by Lagrange and formulated more precisely by Euler is connected to an integral of velocity in space. In any case we will call it Maupertuis principle of least action and we remark that it should no be confused with the Hamilton principle of least action which appeared later But the main result of the theory is the Lagrange equations of motion derived from the fundamental principle.

According to Lagrange, the first principle was stated by Huygens, based on a result of Galileo concerning the final velocities of descending bodies. Johann Bernoulli derived this principle from the laws of motion and gave to it the name of conservation of living forces. Daniel Bernoulli used the principle in his studies of hydrodynamics. The second principle is due to Newton, who showed in his Principles that the state of rest or motion of the center of gravity of several bodies is not changed by reciprocal action of these bodies. The third principle, says Lagrange, seems to be discovered independently by Euler, Daniel Bernoulli and d'Arcy. We should add that the origin of this principle lays in the areal law of Kepler which was demonstrated by Newton in his Principles from his laws of motion applied to central forces. The fourth principle is the one introduced by Maupertuis according to which the sum of the product of the masses with the velocities and the spaces traversed is a minimum. A more precise statement of the principle was given by Euler. Lagrange does not regard this principle as a metaphysical principle as did Maupertuis but as a simple and general result of the laws of mechanics.

The d'Alembert principle was stated in his Treatise on Dynamics [51, published in 1743. According to Lagrange, this treatise provided a general and direct method to equate the problems of dynamics, reducing the laws of motion to those of equilibrium, rendering the dynamics to the statics. D'Alembert conceived his principle by adding to the Johann Bernoulli principle of virtual velocities the so called inertial forces. These are not properly forces in the usual sense. The inertial force of a body is just an abbreviation for the mass multiplied by the acceleration. Lagrange formulates the d'Alembert principle as follows. Let us denote by $P, Q, R$ the forces acting on a material point of mass $m$ along the directions $p, q, r$ and let $x, y, z$ be the rectangular coordinates of this material point at a certain instant of time $t$. The use of rectangular axes fixed in space is a crucial step in Lagrange reasoning because it allows to write the accelerations along these direction as $d^{2} x / d t^{2}, d^{2} y / d t^{2}$, and $d^{2} x / d t^{2}$, the same form for all three directions. He reminds that the use of three rectangular fixed axes was employed by Maclaurin in 1742. The expression of the 
d'Alembert principle is written in the form

$$
\begin{array}{r}
\sum m\left(\frac{d^{2} x}{d t^{2}} \delta x+\frac{d^{2} y}{d t^{2}} \delta y+\frac{d^{2} z}{d t^{2}} \delta z\right) \\
+\sum(P \delta p+Q \delta q+R \delta r)=0
\end{array}
$$

where the summation is over all the material points. Lagrange uses the notation $\delta$ for a generic variation and $d$ for a variation in position resulting from a variation $d t$ in time.

In modern notation, the quantities $m, x, y, z, P, p, Q$, $q, R$, and $r$ appearing after a sing of summation should be labeled by an index, $i$ for instance, which in turn should be indicated in the sign of summation. We also remark that Lagrange uses a capital letter $S$ for the sign of summation instead of the capital Greek letter sigma, used here. Notice in addition that the sense in which the forces $F, Q, R$ are used in equation (85) is such that they decrease the velocities when they are positive. To use these quantities in the usual sense of increasing the velocities, we should replace the plus sign in the second summation by a minus sign. However, we will not do this and stick to Lagrange convention.

From the fundamental principle given by equation (85), Lagrange demonstrates the four laws stated above. The second is derived by first transforming the variables $p, q, r$ into the rectangular variables $x, y, z$ in which case the equation becomes

$$
\begin{gathered}
\sum\left(m \frac{d^{2} x}{d t^{2}}+X\right) \delta x+\sum\left(m \frac{d^{2} y}{d t^{2}}+Y\right) \delta y \\
+\sum\left(m \frac{d^{2} z}{d t^{2}}+Z\right) \delta z=0 .
\end{gathered}
$$

Let us assume that the forces remains unchanged if each one of the variables $x, y$, and $z$ suffer the same translations $a, b$, and $c$, respectively. After performing the change of variables the equation 86 will contain terms in $\delta a, d b$ and $d c$ and, as these variations are independent, their coefficients should vanish leading to the results

$$
\begin{aligned}
& \sum m \frac{d^{2} x}{d t^{2}}+\sum X=0 \\
& \sum m \frac{d^{2} y}{d t^{2}}+\sum Y=0 \\
& \sum m \frac{d^{2} z}{d t^{2}}+\sum Z=0
\end{aligned}
$$

which are the expression of the conservation of the center of gravity.

The third law is demonstrated by considering a rotation of the system about a fixed axis which is accomplished by the following expressions for the variations on $x, y$, and $z: \delta x=z \delta \omega-y \delta \varphi, \delta y=x \delta \varphi-z \delta \psi$, $\delta z=y \delta \psi-x \delta \omega$. Again the coefficients of $\delta \omega, \delta \psi$, and $\delta \varphi$ in equation 85 are independent leading to the equations

$$
\begin{aligned}
& \sum m\left(x \frac{d^{2} y}{d t^{2}}-y \frac{d^{2} x}{d t^{2}}\right)+\sum(x Y-y X)=0 \\
& \sum m\left(z \frac{d^{2} x}{d t^{2}}-x \frac{d^{2} z}{d t^{2}}\right)+\sum(z X-x Z)=0 \\
& \sum m\left(y \frac{d^{2} z}{d t^{2}}-z \frac{d^{2} y}{d t^{2}}\right)+\sum(y Z-z Y)=0
\end{aligned}
$$

which are the expression of the conservation of the motion of rotation.

The first and fourth laws are demonstrated by assuming that the second summation in (85), involving the forces, is an exact differential of a function $V$, that is

$$
d V=\sum(P d p+Q d q+R d r)
$$

In modern terms, $V$ is understood as the potential energy and the forces are said to be conservative. To demonstrate the first law Lagrange replaces the variations $\delta x, \delta y, \delta z$, by the differentials $d x, d y, d z$, which represent the actual distances traversed by the bodies during an element of time $d t$. The equation 85 becomes

$$
\sum m\left(\frac{d^{2} x}{d t^{2}} d x+\frac{d^{2} y}{d t^{2}} d y+\frac{d^{2} z}{d t^{2}} d z\right)+d V=0 .
$$

Next we observe that the summation in (94) is the differential $d T$ of

$$
T=\frac{1}{2} \sum m\left(\frac{d x^{2}}{d t^{2}}+\frac{d y^{2}}{d t^{2}}+\frac{d z^{2}}{d t^{2}}\right) .
$$

from which follows that $d T+d V=0$ or $T+V$ equal to a constant, which is the expression of the conservation of the living forces.

Before deriving the fourth law, we will transform the expression 85 into an equivalent form. To this end we use the identity

$$
\begin{aligned}
& d^{2} x \delta x+d^{2} y \delta y+d^{2} z \delta z \\
& \quad=d(d x \delta x+d y \delta y+d z \delta z)-\frac{1}{2} \delta\left(d x^{2}+d y^{2}+d z^{2}\right)
\end{aligned}
$$

obtained by the usual rules of differentiation and by taking into account that $d$ and $\delta$ may be interchanged, as in $d \delta x=\delta d x$, as argued by Lagrange. Replacing this expression in equation 85 and taking into account the definition of $T$ given by (95), we reach the result

$$
d \sum \frac{m}{d t^{2}}(d x \delta x+d y \delta y+d z \delta z)-\delta T+\delta V=0
$$

and we are considering that the second summation in 85 is an exact differential.

The fourth law, the Maupertuis principle of least action, is derived by considering that the variation in the variables $x, y, z$ are taken only over trajectories such 
that the sum $T+V$ is a constant. From this restriction, it follows that $\delta T+\delta V=0$ which ones uses to replace $\delta V$ in equation (97) by $-\delta T$, which gives the result

$$
d \sum \frac{m}{d t^{2}}(d x \delta x+d y \delta y+d z \delta z)-2 \delta T=0 .
$$

Denoting by $d s$ and element of the trajectory, the velocity is $u=d s / d t$ and $u^{2}=\left(d x^{2}+d y^{2}+d z^{2}\right) / d t^{2}$. Then from the definition (95) of $T$, we have $T=$ $(1 / 2) \sum u^{2}$ and $\delta T=\sum m u \delta u$, which replaced in 98 gives

$$
d \sum m\left(\frac{d x}{d t} \delta x+\frac{d y}{d t} \delta y+\frac{d z}{d t} \delta z\right)-\delta \sum m u d s=0,
$$

after multiplying by $d t$.

Now we integrate this equation along a trajectory such that $T+V$ is a constant with the condition that the initial and final points belong to the actual trajectory. This condition implies that $\delta x, \delta y$, and $\delta z$ vanish at the initial an final points and the integral of the first summation in $(99)$ vanishes and we are left with

$$
\delta \int \sum m u d s=0 .
$$

This is the expression of Maupertuis principle of least action as it had been formulated by Euler for the case of one body [52, 53] and by Lagrange [48, 50]. It should be remarked that the integral between two given points is constrained to the paths such that $T+V$ is a constant.

\section{Equations of motion}

In the fourth section on dynamics, Lagrange derives from 85 his equations of motion. He starts by changing variables from $x, y, z$ to $\xi, \psi, \varphi$ which gives linear relations between $d x, d y, d z$ and $d \xi, d \psi, d \varphi$, and also the same linear relations between $\delta x, \delta y, \delta z$ and $\delta \xi$, $\delta \psi, \delta \varphi$, the coefficients being functions of the new variables. From the linear relations we see that quadratic forms on the differentials of the old variables become quadratic forms on the new variables. Thus from this transformation, we may write

$$
\begin{aligned}
d x \delta x & +d y \delta y+d z \delta z \\
= & A d \xi \delta \xi+B d \psi \delta \psi+C d \varphi \delta \varphi+D d \xi \delta \psi+E d \xi \delta \varphi \\
& +F d \psi \delta \varphi+D d \psi \delta \xi+E d \varphi \delta \xi+F d \varphi \delta \psi,
\end{aligned}
$$

and

$$
\begin{aligned}
d x^{2}+ & d y^{2}+d z^{2}=A d \xi^{2}+B d \psi^{2}+C d \varphi^{2} \\
& +2 D d \xi d \psi+2 E d \xi d \varphi+2 F d \psi d \varphi
\end{aligned}
$$

where $A, B, C, D, E, F$ are functions of the new variables $\xi, \psi, \varphi$. Defining $\alpha=\left(d x^{2}+d y^{2}+d z^{2}\right) / 2$, we find

$$
\begin{aligned}
\frac{\delta \alpha}{\delta d \xi} & =A d \xi+D d \psi+E d \varphi \\
\frac{\delta \alpha}{\delta d \psi} & =B d \psi+D d \xi+F d \varphi \\
\frac{\delta \alpha}{\delta d \varphi} & =C d \varphi+E d \xi+F d \psi
\end{aligned}
$$

Lagrange uses the notation $\delta / \delta$ for partial derivative. The derivatives $\delta \alpha / \delta \xi, \delta \alpha / \delta \psi$, and $\delta \alpha / \delta \varphi$ are obtained from expression 102 by merely deriving the coefficients $A, B, C, D, E$, and $F$ with respect to the variables $\xi$, $\psi$, and $\varphi$, respectively.

Replacing these results in the identity (96), we find

$$
\begin{array}{r}
d^{2} x \delta x+d^{2} y \delta y+d^{2} z \delta z=-\frac{\delta \alpha}{\delta \xi} \delta \xi-\frac{\delta \alpha}{\delta \psi} \delta \psi-\frac{\delta \alpha}{\delta \varphi} \delta \varphi \\
+d\left(\frac{\delta \alpha}{\delta d \xi}\right) \delta \xi+d\left(\frac{\delta \alpha}{\delta d \psi}\right) \delta \psi+d\left(\frac{\delta \alpha}{\delta d \varphi}\right) \delta \varphi
\end{array}
$$

and the first part of the equation 85 becomes

$$
\begin{gathered}
\sum m\left(\frac{d^{2} x}{d t^{2}} \delta x+\frac{d^{2} y}{d t^{2}} \delta y+\frac{d^{2} z}{d t^{2}} \delta z\right)=\left(d \frac{\delta T}{\delta d \xi}-\frac{\delta T}{\delta \xi}\right) \delta \xi \\
+\left(d \frac{\delta T}{\delta d \psi}-\frac{\delta T}{\delta \psi}\right) \delta \psi+\left(d \frac{\delta T}{\delta d \varphi}-\frac{\delta T}{\delta \varphi}\right) \delta \varphi,
\end{gathered}
$$

where $T$ is given by 95 and understood as a function of $\xi, \psi, \varphi$, and $d \xi, d \psi, d \varphi$.

The second term in 85 is written as

$$
\sum(P \delta p+Q \delta q+R \delta r)=\sum(F \delta \xi+G \delta \psi+H \delta \varphi),
$$

by transforming $p, q$, and $r$ into $\xi, \psi$, and $\varphi$.

From the above results, equation 85 is written as

$$
\Xi \delta \xi+\Psi \delta \psi+\Phi \delta \varphi+\ldots=0
$$

where

$$
\begin{aligned}
& \Xi=d \frac{\delta T}{\delta d \xi}-\frac{\delta T}{\delta \xi}+F, \\
& \Psi=d \frac{\delta T}{\delta d \psi}-\frac{\delta T}{\delta \psi}+G, \\
& \Phi=d \frac{\delta T}{\delta d \varphi}-\frac{\delta T}{\delta \varphi}+H,
\end{aligned}
$$

If $\delta V$, is an exact differential, then

$$
F=\frac{\delta V}{\delta \xi}, \quad G=\frac{\delta V}{\delta \psi}, \quad H=\frac{\delta V}{\delta \varphi},
$$

and

$$
\begin{aligned}
& \Xi=d \frac{\delta T}{\delta d \xi}-\frac{\delta T}{\delta \xi}+\frac{\delta V}{\delta \xi}, \\
& \Psi=d \frac{\delta T}{\delta d \psi}-\frac{\delta T}{\delta \psi}+\frac{\delta V}{\delta \psi}, \\
& \Phi=d \frac{\delta T}{\delta d \varphi}-\frac{\delta T}{\delta \varphi}+\frac{\delta V}{\delta \varphi} .
\end{aligned}
$$


If the new variables $\xi, \psi, \varphi, \ldots$ are all independent which means that the variations $\delta \xi, \delta \psi, \delta \varphi$ become indeterminate, then $\Xi=0, \Psi=0, \Phi=0$, which constitute the Lagrange equations of motion. But if it is not the case, that is, if the new variables are not independent, we may use the method of multipliers discussed above to write down the equations of motion.

\section{Hamilton principle of least action}

Let us write equation (97) as

$$
d \sum m\left(\frac{d x}{d t} \delta x+\frac{d y}{d t} \delta y+\frac{d z}{d t} \delta z\right)-\delta(T-V) d t=0
$$

If we integrate this equation along any trajectory with the condition that the initial and final points belong to the actual trajectory, then $\delta x, \delta y$, and $\delta z$ vanish at these two points and the integral of the first summation in 117 vanish and we are left with

$$
\delta \int(T-V) d t=0
$$

Equation 118 is also the expression of a least action principle which should not be confused with the Maupertuis principle of least action. It was not derived by Lagrange in his Analytical Mechanics but was proposed later on by Hamilton in 1834 and for that reason called Hamilton principle of least action.

If the integration in $(118)$ is performed only along trajectories such that $T+V$ is a constant then this equation reduces to

$$
\delta \int 2 T d t=0
$$

which is equivalent to the expression (100) of the Maupertuis principle of least action.

We remark again that the Maupertuis and Hamilton principles of least action are different in the constraints used to perform the procedure of minimization. Both principles minimizes the same integral 118 but one of them, the Maupertuis principle, is subject to a minimization under the restriction that the trajectories fulfill the constraint $T+V$ equal to a constant. We remark in addition that both principles requires that the forces are conservatives. Therefore the Lagrange equations obtained from them are valid only for conservative forces. This is in contrast with the d'Alembert principle from which the Lagrange equations can be derived even for nonconservative forces.

\section{Laplace}

Pierre-Simon Laplace was born in 1749 at Beaumont, in northern France. He attended the Benedictine school of the village from the age of seven to sixteen. In 1766 he was sent to the University of Caen and in 1768 he left to Paris. He was appointed professor of mathematics at the Military School and was elected to the Academy of Sciences in 1773 at the age of twenty-four. In the period of ten years from 1778 he reached most of the major results in the areas of celestial mechanics and probability, which were incorporated into the Treatise on Celestial Mechanics and in the Analytical Theory of Probabilities [56. Laplace also engaged on research on other topics of physics such as the heat capacities of bodies and the related topic of sound velocity. He died in Paris in 1827.

\subsection{Mechanics}

The Treatise on Celestial Mechanics was written in five volumes, divided in fifteen books, and published in the period 1799-1825 [5761]. The treatise contains an exposition of the analytical mechanics and its application to the motion of celestial bodies that attract each other in accordance with inverse square law. Laplace also treats the problem of the shape of celestial bodies, the tidal problem, and develops a perturbation theory to treat the motion of bodies.

Here we will be concerned only with the subjects contained in the first volume, consisting of two books. The first volume, published in 1799, gives an exposition of the laws of mechanics in a form appropriate to be applied to astronomical problems [57. Laplace adopts d'Alembert principle as the fundamental law of mechanics as did Lagrange. In book 1, he derives from this principle the main laws of mechanics for a system of bodies, following reasonings similar to those used by Lagrange, as exposed above. These includes the conservation of living forces, conservation of the motion of the center of gravity, conservation of areas, and the principle of least action.

Denoting by $x, y$, and $z$ the rectangular coordinates of a material point, by $P, Q$, and $R$ the components of the force acting on these directions, Laplace writes the d'Alembert principle for one material point as

$\delta x\left(d \frac{d x}{d t}-P d t\right)+\delta y\left(d \frac{d y}{d t}-Q d t\right)+\delta z\left(d \frac{d z}{d t}-R d t\right)=0$,

where $\delta x, \delta y$, and $\delta z$ are the variations of the rectangular coordinates, and $d x, d y, d z$ increments in these coordinates during an elementary interval of time $d t$. The equations (120) remain valid if one replaces the variations by differentials. Integrating it we get

$$
\frac{d x^{2}+d y^{2}+d z^{2}}{d t^{2}}=c+2 \int(P d x+Q d y+R d z)
$$

where the left hand side is the square of the velocity and $c$ is a constant. If in addition $P d x+Q d y+R d z$ is an exact differential $d \phi$ of a function $\phi$ it may be written as

$$
\frac{d x^{2}+d y^{2}+d z^{2}}{d t^{2}}=c+2 \phi
$$


If the material point is free, the variations are independent and the equations of motion are $d^{2} x / d t^{2}=P$, $d^{2} y / d t^{2}=Q$, and $d^{2} z / d t^{2}=R$. Otherwise, one should employ the relation between the variations to get the equations of motion.

Laplace gives the example of a heavy body moving on a spherical surface. Here we will consider the particular case of this type of motion, which is the motion of a simple pendulum. Denoting by $z$ the vertical coordinate and by $x$ the horizontal coordinate then these variables obeys the constraint $x^{2}+z^{2}=r^{2}$. The method of multipliers gives the equations

$$
\begin{gathered}
d \frac{d x}{d t}+2 \lambda x d t=0 \\
d \frac{d z}{d t}+2 \lambda z d t-g d t=0 .
\end{gathered}
$$

From $x^{2}+z^{2}=r^{2}$, we find $x d x+z d z=0$, and $x^{2} d x^{2}=$ $z^{2} d z^{2}$, which replaced in the relation

$$
\frac{d x^{2}+d z^{2}}{d t^{2}}=c+2 g z
$$

obtained from 122 , we find

$$
r^{2} \frac{d z^{2}}{d t^{2}}=\left(r^{2}-z^{2}\right)(c+2 g z),
$$

and

$$
d t=\frac{-r d z}{\sqrt{\left(r^{2}-z^{2}\right)(c+2 g z)}} .
$$

Performing the change of variables $z=r-h \sin ^{2} \theta$, where $h=c / 2 g+r$, we find

$$
d t=\sqrt{\frac{r}{g}} \frac{d \theta}{\sqrt{1-\gamma^{2} \sin ^{2} \theta}},
$$

where $\gamma^{2}=h / 2 r$. The quarter of the period of oscillation is obtained by integration from $\theta=0$ to $\theta=\pi / 2$. The integration can be performed after the expansion in powers of $\gamma$ with the following result for the period of oscillations

$$
2 \pi \sqrt{\frac{r}{g}}\left[1+\frac{\gamma^{2}}{2^{2}}+\left(\frac{1.3}{2.4}\right)^{2} \gamma^{4}+\left(\frac{1.3 .5}{2.4 .6}\right)^{2} \gamma^{6}+\ldots\right],
$$

a result that had been obtained by Euler [23], as shown by equation 42 . If the oscillations are small then $\gamma$ will be small and it can be neglected. In this case the period of oscillation will be $2 \pi \sqrt{r / g}$.

The book 2 contains the analysis of the motion of celestial bodies based on the inverse squared law of gravitation. Laplace starts by considering the motion of a body under a central and showing that if the trajectory is a conic section and is directed towards the focus then it is proportional to the inverse of the square of the distance of the body to the focus. The demonstration is as follows. The equation of motion of a planet is given by

$$
\frac{d^{2} x}{d t^{2}}+P=0 \quad \frac{d^{2} y}{d t^{2}}+Q=0
$$

where $x$ and $y$ are the rectangular coordinates on the plane of the orbit and $P$ and $Q$ are the forces acting on the planet.

If we sum the first equation multiplied by $-y$ with the second multiplied by $x$, we have

$$
\frac{d(x d y-y d x)}{d t^{2}}+x Q-y P=0 .
$$

It is clear that $x d y-y d x$ is double the are which the radius vector sweeps in the interval of time $d t$. If $Q / P=y / x$, the force is always directed to origin of the coordinate system, that is towards the sun. In this case the area swept by the radius vector is proportional to the time, which is the law of the areas that is,

$$
x d y-y d x=c d t
$$

where $c$ is a constant.

If now we sum the first equation multiplied by $d x$ with the second multiplied by $d y$, we have

$$
\frac{d x d^{2} x+d y d^{2} y}{d t^{2}}+P d x+Q d y=0,
$$

which after integration gives

$$
\frac{d x^{2}+d y^{2}}{d t^{2}}+2 \int(P d x+Q d y)
$$

or, using the law of areas,

$$
\frac{c^{2}\left(d x^{2}+d y^{2}\right)}{(x d y-y d x)^{2}}+2 \int(P d x+Q d y)=0 .
$$

Using new variables $r$ and $\nu$ related to $x$ and $y$ by $x=r \cos \nu$ and $y=r \sin \nu$, and $\phi$, related to $P$ and $Q$ by $P=\phi \cos \nu$ and $Q=\phi \sin \phi$, we find

$$
\begin{gathered}
\frac{c^{2}\left(d r^{2}+r^{2} d \nu^{2}\right)}{r^{4} d \nu^{2}}+2 \int \phi d r=0, \\
d \nu=\frac{c d r}{r^{2} \sqrt{-c^{2} / r^{2}-2 \int \phi d r}}
\end{gathered}
$$

The integration of this equation gives $\nu$ in terms of $r$ if $\phi$ is a function of $r$. That is, it gives the orbit of a planet.

The differentiation of 136 with respect to $r$ gives

$$
\phi=\frac{c^{2}}{r^{3}}-\frac{c^{2}}{2} \frac{d}{d r}\left(\frac{d r^{2}}{r^{4} d \nu^{2}}\right) .
$$

The equation describing a conic section in the variables $r$ and $\nu$ with the origin of the coordinates at the focus is

$$
r=\frac{h}{1+e \cos \nu}
$$


where $b$ is a constant and $e$ is the eccentricity, with $e<1$ for an ellipse, $e=1$ for a parabola and $e>1$ for a hyperbole. From the equation of the conic section we find

$$
\phi=\frac{c^{2}}{h r^{2}}
$$

and the force is inversely proportional to the square of the distance to the focus.

A system of bodies interacting through the mutual attraction given by the inverse squared law is treated by defining a function $\lambda$, which is a sum of terms, each one corresponding to a pair of bodies of the type

$$
\frac{m m^{\prime}}{\sqrt{\left(x^{\prime}-x\right)^{2}+\left(y^{\prime}-y\right)^{2}+\left(z^{\prime}-z\right)^{2}}},
$$

where $m$ and $m^{\prime}$ are the masses, and $x^{\prime}, y^{\prime}, z^{\prime}$, and $x, y, z$ are the rectangular coordinates of the bodies, respectively. In modern terms, $-\lambda$ is the potential energy. The equation of motion of a given body is

$$
\begin{aligned}
& m \frac{d^{2} x}{d t^{2}}=\left(\frac{d \lambda}{d x}\right), \\
& m \frac{d^{2} y}{d t^{2}}=\left(\frac{d \lambda}{d y}\right), \\
& m \frac{d^{2} z}{d t^{2}}=\left(\frac{d \lambda}{d z}\right),
\end{aligned}
$$

where a partial derivative is denoted by enclosing the fraction between parentheses.

From the equations of motion, Laplace shows that the center of mass is at rest or moves with a constant velocity. He shows the law of areas, translated by

$$
\sum m \frac{x d z-z d x}{d t}=c
$$

where $c$ is a constant, and similar equations for $y$ and $z$. He also shows the conservation of living forces,

$$
\sum m \frac{d x^{2}+d y^{2}+d z^{2}}{d t^{2}}-2 \lambda=h
$$

where $h$ is a constant.

Laplace comments that these three laws give seven integrals of motion. If the system is composed by two bodies, these integrals allow the reduction of the problem to to differential equations of the first order, which can thus be integrated. But if the system consists of three or more bodies it is necessary to resort to approximation methods.

Let us consider three bodies. The larger, representing the sun, with mass $M$, and two others representing a planet and a satellite, with masses $m_{0}$, and $m_{1}$, respectively. We denote by $x_{0}, y_{0}, z_{0}$, and $x_{1}, y_{1}, z_{1}$ the rectangular coordinates of the smaller bodies in relation to the larger one. The mass $M$ is large enough to considered the large body at rest. The corresponding function $\lambda$ is

$$
\lambda=\frac{M m_{0}}{r_{0}}+\frac{M m_{1}}{r_{1}}+\frac{m_{0} m_{1}}{r},
$$

where $r_{0}=\sqrt{x_{0}^{2}+y_{0}^{2}+z_{0}^{2}}, r_{1}=\sqrt{x_{1}^{2}+y_{1}^{2}+z_{1}^{2}}$ are the distances of the smaller bodies to the larger one, and $r=\sqrt{x^{2}+y^{2}+z^{2}}, x=x_{1}-x_{0}, y=y-y_{0}, z=z_{1}-z_{0}$.

Denoting the coordinates of the center of mass by $X$, $Y, Z$, its equation of motion is

$$
m \frac{d^{2} X}{d t^{2}}=\left(\frac{d \lambda}{d X}\right)=-\frac{M m_{0}}{r_{0}^{3}} x_{0}-\frac{M m_{1}}{r_{1}^{3}} x_{1},
$$

and similar equations for $Y$, and $Z$, where $m=m_{0}+m_{1}$ is the total mass of the smaller bodies. The equation of motion related to the variables $x, y$, and $z$ are

$$
\mu \frac{d^{2} x}{d t^{2}}=\left(\frac{d \lambda}{d x}\right)=\frac{M \mu}{r_{0}^{3}} x_{0}-\frac{M \mu}{r_{1}^{3}} x_{1}-\frac{m_{0} m_{1}}{r^{3}},
$$

and similar equations for $y$, and $Z z$, where $\mu=$ $m_{0} m_{1} / m$.

Laplace expands $x_{0} / r_{0}^{3}$ and $x_{1} / r_{1}^{3}$ around $X / R^{3}$ with the result

$$
\begin{aligned}
& \frac{x_{0}}{r_{0}^{3}}=\frac{X}{R^{3}}-\frac{m_{1} x}{m R^{3}}+\frac{3 m_{1} X(X x+Y y+Z z)}{m R^{5}}, \\
& \frac{x_{1}}{r_{1}^{3}}=\frac{X}{R^{3}}+\frac{m_{0} x}{m R^{3}}-\frac{3 m_{0} X(X x+Y y+Z z)}{m R^{5}} .
\end{aligned}
$$

From these results one reaches the results

$$
\begin{gathered}
\frac{d^{2} X}{d t^{2}}=\frac{M X}{R^{3}} \\
\frac{d^{2} x}{d t^{2}}=-\frac{m}{r^{3}}-\frac{M x}{R^{3}}+\frac{3 M X(X x+Y y+Z z)}{R^{5}}
\end{gathered}
$$

and similar equations for $Y, Z, y$, and $z$. From the first, we see that the motion of the center of gravity is equivalent to the motion that result if the smaller bodies were reunited at the center of gravity.

In the following, Laplace consider the function $V$ such that $-(d V / d x),-(d V / d y)$, and $-(d V / d z)$ are the components of the force acting on a material point of mass $m$ due to an extended body with density $\rho$. Denoting by $x, y$, and $z$ the rectangular coordinates of the material point then $V$, which in modern terms is the potential, is given by

$$
V=\int \frac{\rho}{R} d x^{\prime} d y^{\prime} d z^{\prime},
$$

where $R=\sqrt{\left(x-x^{\prime}\right)^{2}+\left(y-y^{\prime}\right)^{2}+\left(z-z^{\prime}\right)^{2}}$.

From the definition of $R$ we see that

$$
\frac{d}{d x} \frac{1}{R}=-\frac{\left(x-x^{\prime}\right)}{R^{3}},
$$

and

$$
\frac{d^{2}}{d x^{2}} \frac{1}{R}=-\frac{1}{R^{3}}+\frac{3\left(x-x^{\prime}\right)^{2}}{R^{5}},
$$


and similar equations related to $y$ and $z$. From these equations it follows at once

$$
\frac{d^{2}}{d x^{2}} \frac{1}{R}+\frac{d^{2}}{d y^{2}} \frac{1}{R}+\frac{d^{2}}{d z^{2}} \frac{1}{R}=0 .
$$

Multiplying by $\rho$ and integrating in $x^{\prime}, y^{\prime}$, and $z^{\prime}$, we find

$$
\frac{d^{2} V}{d x^{2}}+\frac{d^{2} V}{d y^{2}}+\frac{d^{2} V}{d z^{2}}=0
$$

which is the Laplace equation. It appeared in a paper by Laplace on the attractions of spheroids, published in 1785 62. Laplace states that it is a remarkable equation and is of great utility in the theory of shapes of celestial bodies. He shows that in spherical coordinates it reduces to the form

$$
\left(\frac{d^{2} V}{d \theta^{2}}\right)+\frac{\cos \theta}{\sin \theta}\left(\frac{d V}{d \theta}\right)+\frac{1}{\sin ^{2} \theta}\left(\frac{d^{2} V}{d \omega^{2}}\right)+r\left(\frac{d^{2} r V}{d r^{2}}\right)=0 .
$$

If the body is a spherical layer then $V$ depends only on $r$ and 159 gives $\left(d^{2} r V / d r\right)=0$ from which follows that $V=A+B / r$, and the force acting on the material point is $-(d V / d r)=B / r^{2}$. If the point is inside, one should consider the solution such that $B=0$ giving a vanishing force. If the point is outside the force is the same as that the mass $M$ of the body were at its center which is $M / r^{2}$ This result remarkable result, says Laplace, is also valid for a sphere consisting of concentric layers and can thus applied to the sun, the planets and satellites, which are very nearly spheres of that type.

In cylindrical coordinates, the Laplace equation 158 reads

$$
r^{2}\left(\frac{d^{2} V}{d r^{2}}\right)+\left(\frac{d^{2} V}{d \omega^{2}}\right)+r\left(\frac{d V}{d r}\right)=0 .
$$

If $r$ is the distance to the axis of a cylinder, the potential $V$ does not depend on $\omega$ and using the notation $F=-(d V / d r)$, the equation 160 becomes

$$
r^{2}\left(\frac{d F}{d r}\right)+r F=0
$$

whose solution is $F=H / r$, where $H$ is a constant.

The rest of the book 2 is devoted to the development of a perturbation theory for a systematic approximation to the motion of celestial bodies.

\subsection{Theory of sound}

According to Newton the velocity of sound in the air is given by $c=\sqrt{p / \rho}$ where $p$ is the pressure and $\rho$ the density. As we have seen above, the value obtained by Newton from this formula by using the experimental values of the pressure and density is one sixth lower than the experimental values of the velocity of sound. The same formula was obtained both by Lagrange and by
Euler by using their theories. They could not explain the discrepancy and Euler, seventy years after Newton, admitted that no one has yet discovered its the cause.

Around the beginning of the nineteenth century, Laplace come up with a new idea that could solve the problem of the discrepancy [8, 63]. This idea led him to state in a publication of 1816 [64], without demonstration, that the velocity of sound is equal to that of Newton multiplied by the square root of the ratio between the specific heat at constant pressure and the specific heat at constant volume. If we denote this ratio by $\gamma$, this is expressed by

$$
c=\sqrt{\gamma p / \rho}
$$

He demonstrated this formula later on in 1822 [65]. As the ratio $\gamma$ is larger than the unity, the velocity of sound is larger than that of Newton and closer to the experimental value. Indeed, in a publication of 1822 , Laplace obtained 332.9 meters per second, by using the value $\gamma=1.354$ for the air and the value 286.1 meters for the velocity of sound at $12.5^{\circ}$ from the Newton formula. The observed value at $7.5^{\circ}$ was 337.2 which corrected by 3.2 gives the observed value of 340.4 meters per second at $12.5^{\circ} 65$.

As the sound involves compressions and rarefactions of the air, the velocity is related to the variation of the pressure $p$ with density $\rho$. A measure of this variation is $d p / d \rho$ and, as we will show below, the velocity of sound is related to this quantity by

$$
c=\sqrt{d p / d \rho} .
$$

However, the calculation of $d p / d \rho$ depends on the thermodynamic process. Laplace argues that the compressions and rarefactions do not occur isothermally but occur without the exchange of heat, or adiabatically. If we denote by $k_{S}$ the value of $d p / d \rho$ calculated along an adiabatic process then according to Laplace $c=\sqrt{k_{S}}$.

Let us denote by $k_{T}$ the value of $d p / d \rho$ calculated along an isothermal process. If the compressions ane rarefactions were isothermal, the velocity of sound would be $c=\sqrt{k_{T}}$. Using the Boyle law, which says that pressure is proportional to density, one finds $k_{T}=p / \rho$, and $c=\sqrt{p / \rho}$, which is the Newton formula. Laplace demonstrated in his publication of 1822 [65] concerning the theory of elastic fluids that the quantities $k_{S}$ and $k_{T}$ are related to the specific heat at constant pressure $c_{P}$ and the specific heat at constant volume $c_{V}$ by

$$
\frac{k_{S}}{k_{T}}=\frac{c_{P}}{c_{V}}
$$

or by $k_{S}=\gamma k_{T}$, as $\gamma$ is the ration of the specific heats, which leads to his formula for the speed of sound in air.

Biot in 1802 [66] and Poisson in 1808 [67] gave derivations of a formula for the velocity of sound which is equivalent to the expression (163). The derivation which we present next follows the Poisson approach, 
which he presented in a paper on the theory of sound, published in 1808 [67]. He uses the equations of motion of a fluid contained in the Analytical Mechanics of Lagrange. Denoting by $\rho$ density and by $u, v$, and $w$, the rectangular velocities at a point $x, y, z$ of the fluid, the first equation reads

$$
\frac{d \rho}{d t}+\frac{d \rho u}{d x}+\frac{d \rho v}{d y}+\frac{d \rho w}{d z}=0
$$

The density as well as the components of the velocity depends on the position $x, y$, and $z$, and on time $t$. This is the Euler continuity equation (46), as we have seen above. The second equation refers to the velocity potential $\phi$ defined by considering that the differential $u d x+v d y+w d z$ so that

$$
u=\frac{d \phi}{d x}, \quad v=\frac{d \phi}{d y}, \quad w=\frac{d \phi}{d z} .
$$

It reads

$$
\int \frac{d p}{\rho}+\frac{d \phi}{d t}+\frac{1}{2}\left(u^{2}+v^{2}+w^{2}\right)=V,
$$

where $p$ is the pressure, considered to be a function of $\rho$, and $V$ is the potential related to the external forces, considered to be conservative, and is the equation 61 considered above. If we derive these equations in relation to $x, y$, and $z$, the equations that result are the hydrodynamic Euler equations (51), (52), and (53). In other terms, if the external forces are conservatives and the flow is irrotational, the integral of these equations is equation 167.

We assume that the air is homogeneous and its density equal to $\rho_{0}$ when the velocities vanish. This density is related to the atmospheric pressure $p_{0}$ by $p_{0}=\rho g h$ where $g$ is the acceleration of gravity and $h$ is the height of homogeneous column of air. For small deviation of the density, we may write $p=p_{0}+k\left(\rho-\rho_{0}\right)$ and $\int d p / \rho=k \xi$ where $\xi=\left(\rho-\rho_{0}\right) / \rho_{0}$, and $k=d p / d \rho$ calculated at $\rho=\rho_{0}$. Up to linear terms in $\xi$ and in the velocities, the two equations (165) and (167) become

$$
\begin{gathered}
\frac{d \xi}{d t}+\frac{d u}{d x}+\frac{d v}{d y}+\frac{d w}{d z}=0, \\
k \xi+\frac{d \phi}{d t}=0 .
\end{gathered}
$$

Replacing 169 in 168 and taking into account 166 we reach the equation

$$
\frac{d^{2} \phi}{d t^{2}}=k \frac{d^{2} \phi}{d x^{2}}+k \frac{d^{2} \phi}{d y^{2}}+k \frac{d^{2} \phi}{d z^{2}} .
$$

From this equation it follows immediately that the sound velocity $c=\sqrt{k}=\sqrt{d p / d \rho}$.

\section{Fourier}

Joseph Fourier was born in 1768 at Auxerre, France. His parents had twelve children and he was the ninth.
In 1780 he entered the École Royale Militaire of Auxerre under the direction of the Benedictine order. After spending the period of 1787-9 at the Abbey Saint Benoit he returned to Auxerre where he taught at his former school. He became directly involved with the Revolution in Auxerre. In 1795 he was appointed professor to the newly founded École Polytechnique. Fourier joined the Egypt expedition in 1798 as scientific adviser and appointed secretary of the Cairo Institute. He returned to France in 1801 and was appointed prefect of the Isère department in the following year, settling in Grenoble. He remained in this city until March 1815 when he left to Lyon and then to Paris, a few months later. He was elected to the Académie des Sciences in 1817 and became its permanent secretary in 1822. He died in 1830 68]. Fourier is best known for developing the series expansion that bears his name. His major achievement in the area of physics is the law of heat conduction and the heat equation, which are the focus of our attention here, and the subject of his Analytical Theory of Heat, published in 1822 69, 70].

The Fourier theory of the propagation of heat is based on the primary concepts of heat and temperature, and on the principle of the communication of heat. The temperature of a body is measured by a thermometer which should be in perfect contact with the body. The scale of a thermometer is set up by assigning the temperature 0 to the melting ice and temperature 1 to the boiling water under the atmospheric pressure. The heat is measured by the ice calorimeter. It is proportional to the mass of ice at temperature 0 which is converted into water at the same temperature. The unit of heat corresponds to the melting of one unit of mass such as one kilogram.

The theory of heat that prevailed in the time of Fourier considered heat as a substance called caloric, the main principle of which was the conservation of heat. Although Fourier did not mention any relation of his conception of heat to that of caloric, he used the conservation of heat in an implicit form. In fact, his theory of heat conduction in solids can be understood as an accomplishment of the caloric theory [71, in view of the conservation of heat.

Although the nature of heat is uncertain, says Fourier, the laws that result from its effects are independent of all hypothesis and can be known. Heat penetrates every substance. When it is not uniformly distributed in a body, it passes from the parts which are more heated to those which are less, and is lost at the surface by radiation. The tendency to reach a uniform distribution and the spontaneous emission at the surface have the effect of changing continuously the temperatures of the different points of the body. The problem of the propagation of heat consists in finding the temperature at each point of the body at a given instant, supposing that the initial temperatures are known. This is accomplished by the solution of the differential equation of heat introduced by Fourier. 
Fourier points out in the introduction of his treatise that the effect of heat cannot be compared to those of an elastic fluid. The motion of heat inside bodies is not of the same character as that of the mechanical motion of elastic particles that describes the sound waves. It would be useless, says Fourier, to attempt to deduce from the hypothesis of mechanics the laws of the propagation of heat. To better understand this point we recall that the analytical theories describing mechanical systems give rise to differential equations that are of the second in time. This is for instance the case of the wave equation that describes the propagation of sound in elastic fluids as given by equation (80). The cause of this conclusion is that the Newton equation of motion, which underlies the mechanical theories, is expressed in terms of the second derivative of space with respect to time. The equation of heat on the other hand, although similar to the wave equation, is of the first order in time and in this sense it could not be derived from the laws of mechanical motion, as claims Fourier.

The fundamental principle of the communication of heat was stated by Fourier as follows. Let us denote by $v$ and $v^{\prime}$ the temperatures of two equal molecules $\mathrm{m}$ and $\mathrm{n}$ separated by a very small distance from each other. If $v^{\prime}$ is larger that $v$, then the quantity of heat that $\mathrm{m}$ receives from $\mathrm{n}$ per unit time, is proportional to the difference $\left(v^{\prime}-v\right)$. To reach the law of of heat conduction, Fourier introduces a second assumption concerning the distribution of temperatures along a homogeneous solid enclose between two parallel planes. If these two planes are maintained at temperatures $a$ and $b$ then, in the stationary regime, the temperature $v$ of a certain point at a distant $z$ from $\mathrm{A}$ is assumed to be linear with $x$,

$$
v=a+\frac{b-a}{e} z,
$$

where $e$ is the length $\mathrm{AB}$ and $a$ is greater than $b$. Using the fundamental principle, the flux of heat $F$ from A to $\mathrm{B}$ is proportional to $(b-a) / e$, that is, $F=K(b-a) / e$ where $K$ is a constant.

Let us consider now two small parallel surfaces inside a solid which differ by a small distance $d z$, and which are small enough so that they may be considered to be plane surface. In this case we may use the formula $F=$ $K(b-a) / e$ which becomes

$$
F=-K \frac{d v}{d z}
$$

where $d v$ is the difference in temperature. The flux of heat $F$ is understood as the quantity of heat per unit time and per unit area. The coefficient $K$ is a measure of the specific conductibility of a substance. The equation 172 is the expression of the Fourier law of heat conduction.

The equation of heat conduction is derived as follows. Let us consider the flow of heat through a slice of thickness $d x$ and area $S$. During an interval of time $d t$, the heat that enter the slice through one of the surfaces, located at $x$, is $K S(d v / d x) d t$ whereas the heat that leaves the slice through the other surface, located at $x+d x$, is $K S(d v / d x) d t+K S\left(d^{2} v / d x^{2}\right) d x d t$. Thus the net flow of heat inside the slice is $K S\left(d^{2} v / d x^{2}\right) d x d t$. This quantity should be equal to the increase in heat in the slice during the interval $d t$, by the conservation of heat.

Denoting the specific heat per unit mass of the substance by $C$ and the density by $D$, then the increase in heat will be the specific heat per unit volume $C D$ multiplied by the volume $S d x$ of the slice and multiplied by the increase $d v$ in temperature. The result is $C D S d x d v$ and we conclude that

$$
\frac{d v}{d t}=\frac{K}{C D} \frac{d^{2} v}{d x^{2}}
$$

which is the heat equation for one dimensional flow. If instead of a slice, we use a small rectangular parallelepiped of volume $d x d y d z$, and consider the flow of heat through its six rectangular faces, we find

$$
\frac{d v}{d t}=\frac{K}{C D}\left(\frac{d^{2} v}{d x^{2}}+\frac{d^{2} v}{d y^{2}}+\frac{d^{2} v}{d z^{2}}\right),
$$

which is the heat equation in three dimensions describing the motion of heat in the interior of solids.

Let us consider a semi infinite plate bounded at the left by the line $x=\pi / 2$, at the right by the line $x=$ $-\pi / 2$, and at the bottom by the line $y=0$. The left and right boundary are maintained at temperature 0 whereas the bottom boundary at temperature 1 . It is required to find the temperature of each point of the plate at the stationary state. In this case one has to solve the stationary heat equation in two dimensions, which is

$$
\frac{d^{2} v}{d x^{2}}+\frac{d^{2} v}{d y^{2}}=0
$$

with the given boundary conditions. Fourier assumes that a solution to this equation is the product $f(x) g(y)$ of two functions, one being a function of $x$ only and the other a function of $y$ only. Replacing the product into the equation 175 w have

$$
\frac{f^{\prime \prime}(x)}{f(x)}+\frac{g^{\prime \prime}(x)}{g(x)}=0 .
$$

We suppose that $f^{\prime \prime}(x) / f(x)=m$ and that $g^{\prime \prime}(x) / g(x)=-m$ where $m$ is a positive constant to be found. From these two equations we find $f(x)=e^{-m x}$ and $g(x)=\cos m y$. The other solution for $f$ is $e^{m x}$ but it should be excluded on account that $f$ cannot become infinite when $x$ grows indefinitely. Taking into account that $g$ should vanish when $y=\pi / 2$, it follows that the possible values of $m$ are $1,3,5$, etc. Therefore a general solution for the temperature is $v=\phi(x, y)$ where

$$
\phi=a e^{-x} \cos y+b e^{-3 x} \cos 3 y+c e^{-5 x} \cos 5 y+\ldots
$$




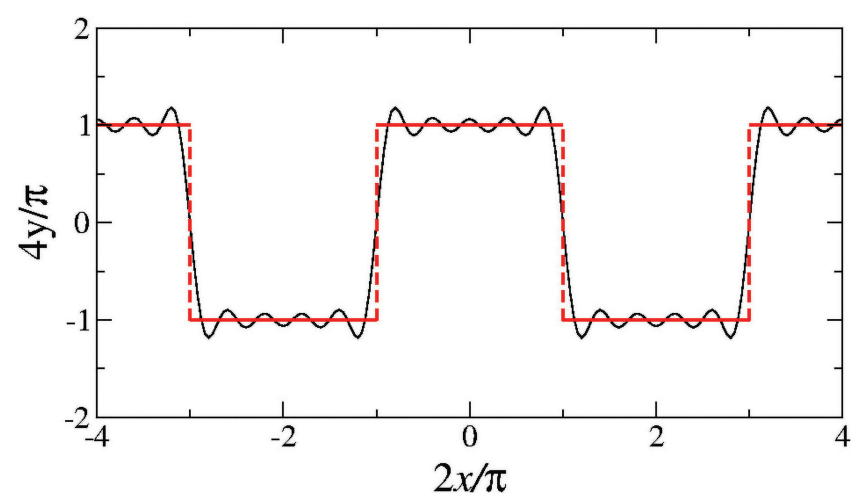

Figure 7: The wavy line represents the Fourier expansion of the square wave given by equation 183 truncated at the tenth term.

It is clear that $\phi(x, \pm \pi / 2)=0$. The other boundary condition that should be fulfilled is $\phi(0, y)=1$, which gives

$$
1=a \cos y+b \cos 3 y+c \cos 5 y+\ldots
$$

and we are left to find the coefficients $a, b, c$, etc.

To find the coefficients, the expression $(178)$ is derived several times after which we set $y=0$. The result is

$$
\begin{gathered}
1=a+b+c+\ldots \\
0=a+3^{n} b+5^{n} c+\ldots
\end{gathered}
$$

which is a set of linear equations for the coefficients. Fourier was able to solve this equation finding the result $a=4 / \pi, b=-4 / 3 \pi, c=4 / 5 \pi$ and so on, which replaced on equation (177), gives the complete solution

$$
\frac{\pi}{4} v=e^{-x} \cos y-\frac{1}{3} e^{-3 x} \cos 3 y+\frac{1}{5} e^{-5 x} \cos 5 y+\ldots
$$

valid for $x$ nonnegative and $y$ between $-\pi / 2$ and $\pi / 2$.

If the values of the coefficients are replace in equation (178), we find

$$
\frac{\pi}{4}=\cos y-\frac{1}{3} \cos 3 y+\frac{1}{5} \cos 5 y+\ldots
$$

Let us write the expression in the right hand side as a function $y(x)$, that is,

$$
y(x)=\cos x-\frac{1}{3} \cos 3 x+\frac{1}{5} \cos 5 x+\ldots
$$

At first sight it seems odd that the function $y(x)$ is a constant $\pi / 4$. However, Fourier warns that $y(x)$ is in fact a function which is understood as being composed by successive segments parallel to the abscissa that takes alternatively the values $\pi / 4$ and $-\pi / 4$ as shown in Figure 7. The exceptions to this rule are the values of $y$ which are odd multiples of $\pm \pi / 2$ in which cases $h$ vanishes. The Figure 7 also shows the plot of the expansion 183 considering only ten terms.

\section{Poisson}

Siméon Denis Poisson was born in 1781 at Pithiviers, France. In 1796 he was enrolled at the École Centrale of Fontainebleau. He was admitted to the École Polytechnique in Paris in 1798, being placed first in the entrance examination, and immediately after his graduation in 1800 he was appointed to a teaching position at the school. In 1802 he became substitute professor and in 1806 full professor. He died in 1840 . Poisson made contributions to mathematics, astronomy, analytical mechanics, theory on sound, theory of heat, optics, electricity and magnetism [72].

\subsection{Electricity}

The theory of electricity of Poisson is contained in two papers concerning the distribution of electricity on the surface of conductor bodies, published in 1812 and 1814, which we analyze here [73, 74]. Poisson assumes that the electric phenomena are attributed to two types of fluids, vitreous and resinous, that extend over all bodies. These electric fluids repel each other if they are of the same type and attract each other if they are of different types. In both cases the force of attraction or repulsion between two molecules of the fluid is assumed to be proportional to the inverse of the square of the distance, in accordance with Coulomb observations, which constitutes the fundamental principle of the theory developed by Poisson.

The main purpose of the theory is the study of the mutual action of electrified conductors placed on dry air so that they do not lose electricity. Poisson remarks that the electric fluid moves to the surface on account of its repulsion and its free motion. The problem remains to find the surface distribution of the fluid in equilibrium.

The approach used by Poisson is based on the use of the function introduced by Lagrange and used by Laplace, which was later called potential function by Green. The relevant property of this function is that the components of the force is obtained by partial differentiation with respect to the space coordinates. As the electric force acting at a certain point at a distant $R$ from a certain molecule is proportional to $1 / R^{2}$, then the corresponding potential function is proportional to $1 / R$. The potential function due to several molecules will be a sum of terms, each one being inversely proportional to the distance of that point to each one of the molecules.

The potential function at a point $\mathrm{A}$ with coordinates $x, y$, and $z$, produced by the electric fluid contained in a certain region of the space is determined as follows. Let us denote by $x^{\prime}, y^{\prime}$, and $z^{\prime}$ the rectangular coordinates of a point $\mathrm{B}$ of that region. The potential function $V$ is

$$
V=\int \frac{\rho d x^{\prime} d y^{\prime} d z^{\prime}}{R},
$$


where $\rho$ is the density of the electric fluid and $R$ is the distance between $\mathrm{A}$ and $\mathrm{B}$ and, given by

$$
R^{2}=\left(x-x^{\prime}\right)^{2}+\left(y-y^{\prime}\right)^{2}+\left(z-z^{\prime}\right)^{2} .
$$

Now the region is supposed to be a very thin spherical shell of radius $a$ and the thickness of the electrical fluid is $\sigma$, which we interpreted as the surface density of the electrical fluid. Using spherical variables defined by $x^{\prime}=r^{\prime} \sin \omega \cos \varphi, x^{\prime}=r^{\prime} \sin \omega \sin \varphi$, and $y^{\prime}=r^{\prime} \cos \omega$, the integral becomes

$$
V=a^{2} \iint \frac{\sigma}{R} \sin \omega d \omega d \varphi
$$

where $\sigma$ depends on the point of the surface, that is, depends on $\omega$ and $\varphi$ and may be of either sign.

Denoting by $r, \theta$, and $\phi$ the spherical coordinates of the point $\mathrm{A}$, that is, $x=r \sin \theta \cos \phi, x=r \sin \theta \sin \phi$, and $y=r \cos \theta$, the distance $R$ is given by

$$
R^{2}=r^{2}+a^{2}-2 r a \cos \gamma
$$

where $\gamma$ is the angle between the lines that connect the points $\mathrm{A}$ and $\mathrm{B}$ to the origin of the reference frame, given by

$$
\cos \gamma=\cos \theta \cos \omega+\sin \theta \sin \omega \cos (\phi-\varphi) .
$$

The method used by Poisson to determine $V$ rests on the expansion of $1 / R$ on powers of $r / a$ or $a / r$ depending whether the point $\mathrm{A}$ is inside or outside the spherical shell. In the first case

$$
\frac{1}{R}=\frac{1}{a} U_{0}+\frac{r}{a^{2}} U_{1}+\frac{r^{2}}{a^{3}} U_{2}+\ldots
$$

and in the second case

$$
\frac{1}{R}=\frac{1}{r} U_{0}+\frac{a}{r^{2}} U_{1}+\frac{a^{2}}{r^{3}} U_{2}+\ldots
$$

where $U_{0}=1$ and $U_{n}$ is a function of $\omega$ and $\varphi$ and fulfills the differential equation

$\frac{1}{\sin \omega} \frac{\partial}{\partial \omega}\left(\sin \omega \frac{\partial U_{n}}{\partial \omega}\right)+\frac{1}{\sin ^{2} \omega} \frac{\partial^{2} U_{n}}{\partial \varphi^{2}}+n(n+1) U_{n}=0$

as shown by Laplace in book 3 of volume 2 of his Celestial Mechanics. Replacing the expansions of $1 / R$ into (186), we obtain similar expansions

$$
V=\frac{1}{a} V_{0}+\frac{r}{a^{2}} V_{1}+\frac{r^{2}}{a^{3}} V_{2}+\ldots
$$

valid inside the shell, and

$$
V=\frac{1}{r} V_{0}+\frac{a}{r^{2}} V_{1}+\frac{a^{2}}{r^{3}} V_{2}+\ldots
$$

valid outside the shell, where the coefficients $V_{n}$ are given by

$$
V_{n}=a^{2} \int \sigma U_{n} \sin \omega d \omega d \varphi
$$

In the case of a surface uniformly electrified, $\sigma$ is a constant, which we set equal to $b$. The point $\mathrm{A}$ is chosen to be at the $z$ axis, which is equivalent to set $R^{2}=$ $r^{2}+a^{2}-2 r a \cos \omega$, and $V_{n}$ vanishes except $V_{0}$ which equals $4 \pi a^{2} b$ from which one finds $V=4 \pi b a$ if the point A is inside the spherical shell and $V=4 \pi b a^{2} / r$ if it is outside.

A relevant result of the mechanics of systems attracting through the inverse square law was demonstrated by Laplace. He showed that the potential $V$ at a point free from matter such as a point outside an extended body or at a point inside a hollow body obeys the equation 158, which is also valid for electric systems as these systems also involve the inverse square law. In fact the result (191) used by Poisson is a consequence of the equation 158). If the point is not free from matter, then the potential does not obey equation 158 but obeys the equation

$$
\frac{d^{2} V}{d x^{2}}+\frac{d^{2} V}{d y^{2}}+\frac{d^{2} V}{d z^{2}}=-4 \pi \rho
$$

which is known as the Poisson equation. In this equation $\rho$ is the mass density, in the case of mechanical systems, or the electrical density, in the case of electrical systems.

The demonstration was given by Poisson in a paper published in 1813 and is as follows [75]. We consider the potential $V$ given by (184) where $R$ is given by (185) The differentiation of $1 / R$, gives

$$
\begin{aligned}
\frac{d^{2}}{d x^{2}} \frac{1}{R} & =\frac{3\left(x-x^{\prime}\right)^{2}-R^{2}}{R^{5}}, \\
\frac{d^{2}}{d y^{2}} \frac{1}{R} & =\frac{3\left(y-y^{\prime}\right)^{2}-R^{2}}{R^{5}}, \\
\frac{d^{2}}{d x^{2}} \frac{1}{R} & =\frac{3\left(z-z^{\prime}\right)^{2}-R^{2}}{R^{5}} .
\end{aligned}
$$

If we sum these three quantities, the result vanishes as long as $R$ is nonzero, that is, as long as the point $\mathrm{B}$ with coordinates $x^{\prime}, y^{\prime}, z^{\prime}$ does not coincide with the point $\mathrm{A}$ with coordinates $x, y, z$, which occurs if $\mathrm{A}$ is outside the region of integration.

Let us see what happens when $\mathrm{A}$ is inside the region of integration. In this case we split the integral 184 in two parts. One of them contains the point $\mathrm{A}$ and the other does not. Denoting the first integral by $U$ then

$$
\frac{d^{2} U}{d x^{2}}=\int \rho \frac{d^{2}}{d x^{2}} \frac{1}{R} d x^{\prime} d y^{\prime} d z^{\prime}
$$

As the region of integration around the point $\mathrm{A}$ can be chosen at will, we choose it to be a very small region, in which case the density can be considered to be the same in all points inside the region and equal to the density at the point $\mathrm{A}$, and the integral becomes

$$
\frac{d^{2} U}{d x^{2}}=\rho \int \frac{d^{2}}{d x^{2}} \frac{1}{R} d x^{\prime} d y^{\prime} d z^{\prime}
$$


Therefore

$$
\frac{d^{2} U}{d x^{2}}+\frac{d^{2} U}{d z^{2}}+\frac{d^{2} U}{d z^{2}}=\rho, I
$$

where $I$ is the integral

$$
I=-\int\left(\frac{d^{2}}{d X^{2}} \frac{1}{R}+\frac{d^{2}}{d Y^{2}} \frac{1}{R}+\frac{d^{2}}{d Z^{2}} \frac{1}{R}\right) d X d Y d Z
$$

obtained by the change of variables $X=x^{\prime}-x, Y=y^{\prime}-$ $y$, and $Z=z^{\prime}-z$, where here $R^{2}=X^{2}+Y^{2}+Z^{2}$, and the integration is performed inside a sphere. The integration gives $I=-4 \pi$, a result independent of the radius of the sphere, and we reach the Poisson equation (195).

\subsection{Magnetism}

Poisson presented his theory of magnetism in two papers published in 1826 [76, 77]. His theory is based on the assumption that the magnetic phenomena are attributed to two types fluids, austral and boreal. The two types of magnetic fluids repel each other if they are of the same kind and attract each other if they are of distinct type. The force is assumed to be proportional to the inverse of the square of the distance between two elements of a magnetic fluid, in accordance with Coulomb observations. In this sense magnetism is analogous to electricity but there are significant differences. Whereas the electricity fluid can pass freely from a conductor to another when they are in contact, the magnetic fluid cannot be transported from a piece of iron to another. The two types of fluids, boreal and austral, that a piece of iron hold in its natural state, only suffers small displacement inside the body when they are separated from external action, such as the induction by a magnet.

Poisson assumes that a magnetic body, such as a piece of iron or nickel, is made up of a collection of small magnetic elements, well separated from one another, each one consisting of equal quantities of austral and boreal fluids located at a thin layer of their surfaces. The magnetic elements are the only places where the magnetic fluids can be found. The process of magnetization corresponds to the separation of the two fluid within each magnetic element and this state of magnetization is maintained by the coercive force that opposes a further separation or the approach of the two fluids.

The main problem is to determine the resultant of the forces, in strength and direction, exerted by a magnetic body on a point $\mathrm{A}$, or the potential at point $\mathrm{A}$ as the forces can be obtained from the potential. As the magnetic body consists of magnetic elements, it is necessary first to determine the potential at $\mathrm{A}$, with coordinates $x, y$, and $z$, due to a magnetic element located within the magnetic body at the point B, with coordinates $x^{\prime}, y^{\prime}$, and $z^{\prime}$. To this end we consider a point $\mathrm{C}$ located at the surface of the magnetic element $\mathrm{B}$. The potential at $\mathrm{A}$ due to the point $\mathrm{C}$, denoted by $q$, is inversely proportional to the distance $R_{1}$ from $\mathrm{C}$ to $\mathrm{A}$, and is given by

$$
q=\int \frac{\sigma}{R_{1}} d s^{\prime \prime},
$$

where $\sigma$ is the surface density of magnetic fluid, and the integration is over the surface of the magnetic element.

Taking into account that the distance of a point of the surface to the center B of the magnetic element is small compared to the distance $R$ from $\mathrm{B}$ to $\mathrm{A}$, we may expand $1 / R_{1}$ around $1 / R$. The first term of the expansion is

$$
\frac{1}{R} \int \sigma d s^{\prime \prime}
$$

and vanishes because the amount of boreal and austral fluids in a magnetic element are equal. The result up to the first order is

$$
q=\alpha \frac{X}{R^{3}}+\beta \frac{Y}{R^{3}}+\gamma \frac{Z}{R^{3}},
$$

where $X=x-x^{\prime}, Y=y-y^{\prime}$, and $Z=z-z^{\prime}$, and $R^{2}=$ $X^{2}+Y^{2}+Z^{2}$, and $\alpha, \beta$, and $\gamma$ are surface integrations of $\sigma$, where the element of area is the projection to a plane perpendicular to the $x, y$, and $z$ directions, respectively. These three quantities determine how the magnetic fluids are distributed within the magnetic element at B. Poisson remarks that a magnetic element is equivalent to a small magnetic needle and that $\alpha, \beta$, and $\gamma$ are understood as proportional to the cosines of the angles defining the axis of the needle.

The potential $Q$ at point A due to the magnetic body is obtained by summing over all magnetic elements of the body

$$
Q=\int k q d x^{\prime} d y^{\prime} d z^{\prime}
$$

where $k$ is number of magnetic elements per unit volume, which we consider to be constant. If $\alpha, \beta$, and $\gamma$ are independent of $x^{\prime}, y^{\prime}$, and $z^{\prime}$, then $q$ can be written as

$$
q=\frac{d}{d x^{\prime}} \frac{\alpha}{R}+\frac{d}{d y^{\prime}} \frac{\beta}{R}+\frac{d}{d z^{\prime}} \frac{\gamma}{R}
$$

and the integral 206 can be transformed into the surface integral

$$
Q=k \int(\alpha \lambda+\beta \mu+\gamma \nu) \frac{d \sigma^{\prime}}{R},
$$

where $\lambda, \mu$, and $\nu$ are the cosines of the angles that defines the direction of the normal to surface of the body.

Let us apply the results to a magnetic body which is a sphere of radius $a$. We choose the frame of reference such that the point $\mathrm{A}$ is at the $z$ axis, which is equivalent to say that $R^{2}=r^{2}+a^{2}-2 r a \cos \omega$, where $\omega$ is the polar angle. In addition we set the axis of the magnetic element perpendicular to the $y$ axis in which case $\beta=0$, and $\alpha^{2}+\gamma^{2}=1$. The potential $Q$ is thus

$$
Q=k \int \frac{1}{R}(\alpha \sin \omega \cos \varphi+\gamma \cos \omega) a^{2} \sin \omega d \omega d \varphi
$$


Before performing the integral Poisson expands $1 / R$ in powers of $1 / r$. After the integration, the only surviving terms is the one that corresponding to $1 / r^{2}$, with the result

$$
Q=\frac{4}{3} \pi a^{3} h \frac{\cos \theta}{r^{2}}
$$

where we have replaced $k \gamma$ by $h \cos \theta$ because $\gamma$ is proportional to the cosine of $\theta$, the angle between the axis of the magnetic elements and the line connection the center of the sphere and the point $\mathrm{A}$.

Below we write down the potential between two magnetic elements characterized by the two sets of quantities $\alpha, \beta, \gamma$, and $\alpha^{\prime}, \beta^{\prime}, \gamma^{\prime}$, which are proportional to the director cosines, respectively. From (207), it is possible to derive the expression for the potential

$$
\begin{aligned}
Q= & \frac{1}{R^{3}}\left(\alpha \alpha^{\prime}+\beta \beta^{\prime}+\gamma \gamma^{\prime}\right) \\
& -\frac{3}{2 R^{5}}(X \alpha+Y \beta+Z \gamma)\left(X \alpha^{\prime}+Y \beta^{\prime}+Z \gamma^{\prime}\right) .
\end{aligned}
$$

The components of the force between the two magnetic elements are determined by differentiation with respect to $x, y$, and $z$. We remark that Poisson did not present this result in his two papers on magnetism but it follows straightforwardly from his theory. In modern terms, the expression (211) is the potential of two magnetic dipoles.

\section{Ampère}

André-Marie Ampère was born in 1775 in Lyon, France. When he was seven years of age, his family moved to Poleymieux, near Lyon. He was not sent to a formal institution education but educated himself at home. In 1802, he was appointed professor of physics at Bourg. He moved to Paris in 1804 when he was appointed assistant professor at the École Polytechnique. He was one of the last to get a teaching position before the educational degrees was required. He was promoted to professor in 1815 and teached continuously at that school until 1828. In 1824, he became professor of physics at the Collège de France. He died in 1836 [78].

The main field of research of Ampère was on electric currents and its magnetics effects, for which he coined the term electrodynamics. He started to work on this subject in 1820, just after the discovery by Oersted that a bar magnet was affected by electrical currents. His investigations and his theory on this subject are found in his Theory of Electrodynamic Phenomena [79], published in 1826 [79]. He also worked on mathematics and chemistry and was one of the supporters of the law of Avogadro, published in 1811, stating that equal volumes of gases at the same temperature and pressure contain the same number of molecules. In fact he also reached this law independently [8], presented in a publication of 1814 .

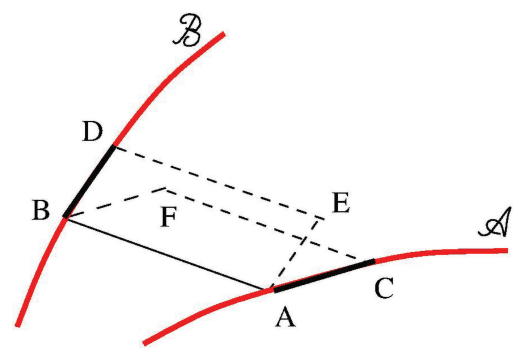

Figure 8: The wires $\mathcal{A}$ and $\mathcal{B}$ carry electrical currents $I$ and $I^{\prime}$ respectively. The length of $\mathrm{AC}$ is $d s$, of $\mathrm{BD}$ is $d s^{\prime}$, the angle $\mathrm{CAB}$ is $\theta^{\prime}$, the angle $\mathrm{ABD}$ is $\theta$, and the angle CAE or FBD is $\gamma$.

\subsection{Electrodynamics}

In 1820, a remarkable discovery was made by Oersted. In July of that year he showed that the orientation of a suspended bar magnet was affected by electric currents [78, 80. Just after being aware of the Oersted results, Ampère himself conducted experiments on the action of electric currents, not on magnets but rather in other electric currents. He showed that two parallel wires carrying electric currents attracted or repelled each other depending whether the currents were in the same direction or in opposite directions, announced in September 1820 [78, 80]. In the following, Ampère sought to determine the law of force between two elements of wires carrying currents 80 . The action between the two electric currents he called electrodynamic phenomenon whereas that between a magnet and an electric current he called electromagnetic phenomenon. At the end of 1820, Ampère arrived at his fundamental formula for the mutual force between two wire elements [78. His formula contained a constant $k$ which only later on, in 1822 , he set to be $k=-1 / 2$ [78].

To properly state the fundamental formula of Ampère, let us consider two wires $\mathcal{A}$ and $\mathcal{B}$ carrying currents $I$ and $I^{\prime}$, respectively, as shown in Figure 8 Denoting by $d s$ the length of an element of the wire $\mathcal{A}$ at a point $\mathrm{A}$ and by $d s^{\prime}$ that of an element of the wire $\mathcal{B}$, at a point $\mathrm{B}$, the force between the two elements, acting along $\mathrm{AB}$, is inversely proportional to the square of the distance $R$ between $\mathrm{A}$ and $\mathrm{B}$, and is given by

$$
I I^{\prime} f d s d s^{\prime},
$$

where

$$
f=\frac{1}{R^{2}}\left(\sin \theta \sin \theta^{\prime} \cos \omega+k \cos \theta \cos \theta^{\prime}\right),
$$

where the constant $k=-1 / 2, \theta$ and $\theta^{\prime}$ are the angles that the current elements $\mathrm{AC}$ and $\mathrm{BD}$ make with the line $\mathrm{AB}$, respectively, and $\omega$ is the angle between the two planes $\mathrm{ABDE}$ and $\mathrm{ABFC}$ containing the line $\mathrm{AB}$, and the current elements $\mathrm{BD}$ and $\mathrm{AC}$, respectively, as shown in Figure 8

Let $\gamma$ be the angle DBF which is equal to the angle EAC and understood as the angle between the two 
current elements. The angles $\theta, \theta^{\prime}$, and $\omega$ are related to $\gamma$ by $\cos \gamma=\sin \theta \sin \theta^{\prime} \cos \omega+\cos \theta \cos \theta^{\prime}$. Using this relation, the formula 213 can written as

$$
f=\frac{1}{R^{2}}\left(\cos \gamma+h \cos \theta \cos \theta^{\prime}\right),
$$

where $h=k-1$ or $h=-3 / 2$. The expression (213) with $k=-1 / 2$, or its equivalent form 214 with $h=-3 / 2$, is the fundamental formula of the electrodynamic theory of Ampère, and usually called Ampère law force.

Let $x, y$, and $z$ be the coordinates of the point $\mathrm{A}$ and $x^{\prime}, y^{\prime}$, and $z^{\prime}$ be the coordinates of the point B. Then $R^{2}=X^{2}+Y^{2}+Z^{2}$, where $X=x-x^{\prime}, Y=y-y^{\prime}$, and $Z=z-z^{\prime}$. The following results can be obtained for the cosines of the angles $\theta, \theta^{\prime}$, and $\gamma$,

$$
\begin{aligned}
& \cos \theta=\frac{d R}{d s} \quad \cos \theta^{\prime}=-\frac{d R}{d s^{\prime}}, \\
& \cos \gamma=-\frac{d R}{d s} \frac{d R}{d s^{\prime}}-R \frac{d^{2} R}{d s d s^{\prime}} .
\end{aligned}
$$

Replacing these results into equation (214), it reads

$$
f=-\frac{1}{R^{2}}\left(R \frac{d^{2} R}{d s d s^{\prime}}-\frac{1}{2} \frac{d R}{d s} \frac{d R}{d s^{\prime}}\right)
$$

which may be transformed into

$$
f=-\frac{1}{\sqrt{R}} \frac{d}{d s^{\prime}}\left(\frac{1}{\sqrt{R}} \frac{d R}{d s}\right) .
$$

We determine now the total force acting between the two circuits $\mathcal{A}$ and $\mathcal{B}$ and consider the case where the two circuits are planar figures belonging to the same plane. The appropriate way to do determine the total force is to sum separately each rectangular component of the force. The component along the $x$ direction is obtained by integrating $(X / R) f$ in $s^{\prime}$ along the circuit $\mathcal{B}$ and in $s$ along the circuit $\mathcal{A}$, that is,

$$
F_{x}=\int f_{x} d s \quad f_{x}=\int \frac{X}{R} f d s^{\prime} .
$$

We remark that the actual forces are obtained by multiplying the above results by $I I^{\prime}$. Replacing the expression 218 for $f$ into equations (219), and after appropriate integrations by parts we find

$$
\begin{gathered}
f_{x} d s=\frac{1}{2} C d y \\
C=\int \frac{1}{R^{3}}\left(X d y^{\prime}-Y d x^{\prime}\right) .
\end{gathered}
$$

Let us consider the case of two parallel straight wires. The wire $\mathcal{A}$ coincides with the $y$ axis and the other, the wire $\mathcal{B}$, is parallel to the first at a distance $\ell$. Then

$$
C=\ell \int \frac{d y^{\prime}}{R^{3}}=\ell \int \frac{d \xi}{\left(\ell^{2}+\xi^{2}\right)^{3 / 2}}=\frac{2}{\ell} .
$$

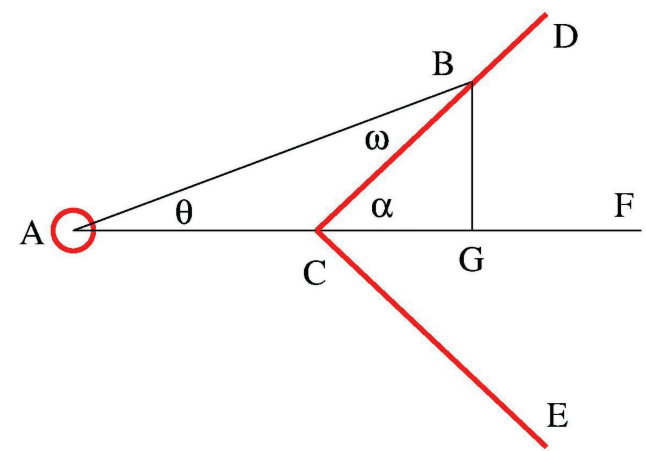

Figure 9: The line ECD is a bended wire carrying an electric current. The distance from its vertex $C$ to the ring at $A$ is $\ell$. The distances $\mathrm{BG}, \mathrm{AG}$ and $\mathrm{AB}$ are $x, y$ and $r$, respectively. If the ring is replaced by a magnet, then the figure also illustrates the arrangement of the Biot experiment.

But $d s=d y$, and we find $f_{x}=1 / \ell$, that is, the force per unit length between the wires is inversely proportional to distance between them.

If the size of the wire $\mathcal{B}$ is small compared to its distance to the wire $\mathcal{A}$, we may expand $1 / R^{3}$ in powers of $x^{\prime}$ and $y^{\prime}$. Up to linear terms in $x^{\prime}$ and $y^{\prime}$, we find

$$
\frac{1}{R^{3}}=\frac{1}{r^{3}}+\frac{3}{r^{5}}\left(x x^{\prime}+y y^{\prime}\right)
$$

where $r=\sqrt{x^{2}+y^{2}}$, and the integral becomes

$$
C=\frac{1}{r^{3}} \int\left(y^{\prime} d x^{\prime}-x^{\prime} d y^{\prime}\right)+\frac{3}{r^{5}} \int\left(x^{2} x^{\prime} d y^{\prime}-y^{2} y^{\prime} d x^{\prime}\right) .
$$

For the case where the wire $\mathcal{B}$ is a small circle of area $a$, we find $C=a / r^{3}$, and

$$
f_{x} d s=\frac{a}{2 r^{3}} d y
$$

We consider now the problem illustrated in Figure 9 which was treated by Savary [81]. The $x$ component of the force on the ring is

$$
F_{x}=a \int \frac{d y}{r^{3}}
$$

where the integral is performed along the upper arm of the wire for which $y=(x-\ell) \tan \alpha$ and we recall that $r=\sqrt{x^{2}+y^{2}}$. Let $\omega$ be the angle ABC between the wire and the line joining the point $\mathrm{B}$ to the center of the ring. The following relations involving this angle are valid: $r \sin \omega=\ell \sin \alpha$ and $r \cos \omega=x \cos \alpha+y \sin \alpha$. Using these relations we change the variable of integration from $y$ to $\omega$, obtaining

$$
F_{x}=\frac{a}{\ell^{2} \sin \alpha} \int \sin \omega d \omega
$$

where the integration runs from $\omega=0$ to $\alpha$. The result is

$$
F_{x}=\frac{a}{\ell^{2} \sin \alpha}(1-\cos \alpha)=\frac{a}{\ell^{2}} \tan \frac{\alpha}{2},
$$


and the force on the ring is inversely proportional to the square of the distance and proportional to $\tan \alpha / 2$.

The arrangement of the ring and the bended wire shown in Figure 9 is such that there is no torque on the ring although there is a net force, which was calculated above. Let us suppose now that the bended wire is rotated ninety degrees around its axis, coinciding with the $x z$ plane. The ring remains in the $x y$ plane. In this configuration there will be no net force on the ring but it will suffer a torque which we will determine in the following. The torque $T$ around the $x$ axis is obtained by multiplying $y^{\prime}$ by the $z$ component of the force, $(Z / R) f$, and integrating in both circuits. That is,

$$
T_{x}=\int \tau_{x} d s \quad \tau_{x}=\int y^{\prime} \frac{Z}{R} f d s^{\prime} .
$$

Taking into account that the ring is small compared to the distance to the bended wire, we expand the integrand of the second integral up to linear terms in $x^{\prime}$ and $y^{\prime}$. We find

$$
\tau_{x} d s=\frac{a z}{2 r^{5}}\left[\left(2 r^{2}-3 x^{2}\right) d x-3 x z d z\right]
$$

where $a=-\int y^{\prime} d x^{\prime}$, which is the area of the ring. Replacing this result in the first integral of $(229)$, and after appropriate integrations by parts, we find the following expression for the torque on the ring

$$
T_{x}=a \int \frac{1}{r^{3}}(x d z-z d x),
$$

where the integral is carried out along the upper arm of the bended wire which is described by $z=\tan \alpha(x-\ell)$. Using this relation, we find

$$
T_{x}=a \ell \int \frac{1}{r^{3}} d z
$$

which can also be written in the form

$$
T_{x}=a \int \frac{\sin \omega}{r^{2}} d s
$$

by the use of the relation $d z=d s \sin \alpha$. Employing the same reasoning used above, we reach the result

$$
T_{x}=\frac{a}{\ell} \tan \frac{\alpha}{2}
$$

and we conclude that the torque is inversely proportional to the distance $\ell$ and proportional to $\tan \alpha / 2$, that is proportional to the tangent of half the inclination of the upper arm in relation to the axis of the bended wire. This result was obtained by Savary and published in 1823 [81].

The electrodynamic theory of Ampère concerned the action between electric currents. But from the results of the theory, some of which we have just obtained, one realizes that a circular current acts on another current like a magnet. This observation lead Ampère to propose that any magnet may be replaced, without change the effects, by a collection of closed electric circuits around the particles of the magnet. In other terms the electric currents of a closed circuit explains the effects of the two fluids called austral and boreal. Ampère advanced the idea that the terrestrial magnetism is produced by electrical currents in the interior of the terrestrial globe.

Ampère drew also the following conclusions concerning the relation of his theory and the Poisson theory of magnetism. The action of a solenoid is the same as that of a series of the Poisson magnetic elements of the same intensity, distributed uniformly along the line that encloses all the small circuits of the solenoid. Incidentally, the term solenoid was created by Ampère in his treatise of 1826 . The action of a closed circuit carrying a electric current is precisely that exerted by the Poisson magnetic elements of the same intensity, distributed uniformly on the surface wrapped up by this circuit.

\subsection{Biot experiments}

The Oersted experiment prompt Biot and Savart to conduct their own experiments on the effect of electric currents on a small magnetized needle, the results of which were presented to the Académie des Sciences in October 1820 [82. They suspended a small magnetized needle which could rotate freely, and placed it in the presence of a straight line wire conducting an electrical current. They observed that the needle turned into a transverse position in relation to the wire in accordance with the Oersted observations. That is, the needle became perpendicular to the plane contained the wire and the point where the needle was placed. In addition they determined the force on the needle and concluded that it was inversely proportional to the distance from the needle to the wire. In fact, the net force on the needle vanishes. What they determined was the magnitude of the two opposite forces acting on the needle, which create a torque on the needle.

When perturbed, the needle performed torsional vibration due to the torque of the opposite forces acting on the needle by the electric current. This allowed them to determine the torque as this quantity is inversely proportional to the square of the period of oscillation. They concluded from the measurements of the period of oscillation that the torque is inversely proportional to the distance from the needle to the wire and so is the magnitude of the two opposite forces.

According to Biot, Laplace showed that their results could be derived by assuming that each small element of electric current exerted a force on the needle which is inversely proportional do the square of the distance [80, 83. The force acting on the needle is obtained by summing over all elements of the wire. Let us suppose that the straight wire coincides with the $y$ axis and that the needle is placed on the $x$ axis, perpendicularly to the plane $x y$, at a distance $\ell$ from the wire. The distance 
from an element $d y$ to the needle is $R=\sqrt{\ell^{2}+y^{2}}$ and, according to Laplace proposition, the force is proportional to $d y / R^{2}$. Integrating over all the element of the wire,

$$
\int \frac{d y}{R^{2}}=\int \frac{d y}{\ell^{2}+y^{2}}=\frac{\pi}{\ell}
$$

that is, the force inversely proportional to $\ell$.

To determine the force for configurations of the wire other than a straight line, Biot considered a bended wire in the form of a V, as shown in Figure 9. The needle is placed at a distance $\ell$ from the vertex of the wire as shown in Figure 9. From the experimental results, Biot found that the force was inversely proportional to $\ell$ and proposed that it was proportional to angle of inclination $\alpha$ [84]. This result was criticized by Ampère who argued, based on a result by Savary, that we have analyzed above, that the force should vary as $\tan (\alpha / 2)$ and not as $\alpha$ [80. Later on, the $\tan (\alpha / 2)$ result was verified experimentally by Biot himself [85. Biot stated that this result could be obtained theoretically by assuming that the force acting of the needle at a distance $R$ from an element of current is proportional to $\sin \omega / R^{2}$, where $\omega$ is the angle between the direction of the element of current and the line joining it to the needle 85 .

\section{Fresnel}

Augustin Fresnel was born in 1788 at Broglie, in northern France. In 1794, his family retired to Mathieu, near Caen. At twelve he was sent to the École Centrale in Caen. He was accepted to the École Polytechnique in 1804 where he spent two years after which he was enrolled at the École des Ponts et Chaussées for three years. After completing his formal training, he entered at the service of the Corps des Ponts et Chaussées as a civil engineer. He died in 1827 86. His researches were confined almost exclusively with optics. By around 1814, he began to consider the wave hypothesis of light. Returning to Mathieu, he undertook experiments on diffraction which confirmed his belief on the wave nature of light. It seems that he reached this idea independently of previous works on the subject such as that of Huygens and Young [86]. To explain the polarization of light, he postulated the concept of transverse waves for light based on a similar proposition by Young.

\subsection{Diffraction}

Here we will examine his memoir on the diffraction of light which won the prize of the Académie des Sciences for the year 1819 and published in 1826 [87. Fresnel adopts the viewpoint that light is a result of vibrations of a universal fluid, called ether, following in this matter Descartes, Hook, Huygens and Euler. It has advantages of offering the explanation of a multitude of optical phenomena which are difficult to be explained by the Newton emission theory.

The Fresnel theory is based on two fundamental principles. The first is the Huygens principle which is stated by Fresnel as follows: The vibrations of a luminous wave in each of its points can be regarded as the sum of the elementary motions that all the parts of this wave, considered in any of its previous positions, and in isolation, would send to these points at the same time [87. The second is the interference principle, which Fresnel state as follows: Two undulations which cross at a small angle are in opposition and weakened when the expanded nodes of one correspond to the condensed nodes of the other, and mutually strengthen each other, on the contrary, when their movements are in harmony [88. According to Fresnel, the interference principle was introduced in optics by Young. However he considered only the extreme cases of agreement or complete discordance between two waves whereas Fresnel considered the intermediate cases and applied it to any number of waves.

A light wave is conceived by Fresnel as the vibrations of the ether substance as much a sound wave is understood as the oscillations of the molecules of the air. The force acting on an ethereal particle is assumed to be proportional do its displacement from equilibrium, resulting in a oscillation of the particle. The solution of the equation of motion gives a sinusoidal behavior for the velocity of the particle which Fresnel writes $u=a \sin 2 \pi t$ by considering the unit of time as being the period of the oscillation. Here, however, we write more generically $u=a \sin \omega 2 \pi \nu t$, where $\nu$ is the reciprocal of the period. To determine the velocity of a particle at a time $t$ and at a distance $x$ from the source of the movement, Fresnel argues that it is the same as that occurring at the instant $t-x / \nu \lambda$ where $\lambda$ is the light wavelength. Thus, the velocity is

$$
u=a \sin 2 \pi\left(\nu t-\frac{x}{\lambda}\right),
$$

where $a$ is called by Fresnel the intensity of vibrations.

Let us consider several waves that reach a given point P located at distances $x, x^{\prime}, x^{\prime \prime}, \ldots$ from the source. By the interference principle, the velocity of a particle at this point is

$$
u=a \sin 2 \pi\left(\nu t-\frac{x}{\lambda}\right)+a^{\prime} \sin 2 \pi\left(\nu t-\frac{x^{\prime}}{\lambda}\right)+\ldots
$$

which can be written as

$$
\begin{gathered}
u=B \sin 2 \pi\left(\nu t-\frac{x}{\lambda}\right)-C \cos 2 \pi\left(\nu t-\frac{x}{\lambda}\right), \\
B=a+a^{\prime} \cos 2 \pi \frac{\ell^{\prime}}{\lambda}+a^{\prime \prime} \cos 2 \pi \frac{\ell^{\prime \prime}}{\lambda}+\ldots \\
C=a^{\prime} \sin 2 \pi \frac{\ell^{\prime}}{\lambda}+a^{\prime \prime} \sin 2 \pi \frac{\ell^{\prime \prime}}{\lambda}+\ldots
\end{gathered}
$$

where $\ell^{\prime}=x^{\prime}-x, \ell^{\prime \prime}=x^{\prime \prime}-x, \ldots$ are the differences in distance of the $\mathrm{P}$ from the sources in relation to one of them. 


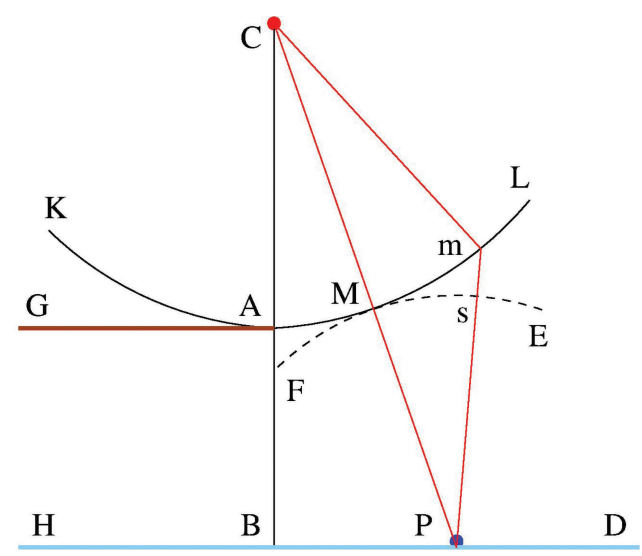

Figure 10: The point $C$ is a source of light waves that is partially intercepted by the opaque body GA. Two trajectories from $C$ to a point $P$ of the screen. Fringes are produced at the screen and are explained by the difference in the lengths of the two paths. The geometric shadow occurs at the left of point $B$

If we define $A$ and $n$ by the relations $B=A \cos 2 \pi n / \lambda$ and $C=A \sin 2 \pi n / \lambda$, the expression (238) becomes

$$
u=A \sin 2 \pi\left(\nu t-\frac{x}{\lambda}-n\right),
$$

and the several waves can be understood as a single wave with intensity of vibrations equal to

$$
A=\sqrt{B^{2}+C^{2}} .
$$

The terms of the right-hand sides of 239 and 240 can be interpreted, following Fresnel, as the rectangular components of forces, or vectors in modern terminology, with intensities $a, a^{\prime}, a^{\prime \prime}$. Using this interpretation, we see that $B$ and $C$ are the rectangular components of the sum of these vectors and that $A$ is the intensity of the sum of the vectors.

The above expressions, which are derived from the interference principles, are applied along with the Huygens principle to the phenomena of diffraction. Here we analyze the diffraction fringes produced by a straight edge as shown in Figure 10 . Let $\mathrm{C}$ be a luminous point understood as a source of waves which are partly intercepted by an opaque body GA. The arc KAML represents the wave at the moment it reaches the edge of the opaque body. According to the Huygens principle we may consider the points at the arc AML as the sources of waves that reach the points of the screen HBD. It is not necessary to consider the points of the arc KA because this part of the wave is reflected by the opaque body.

Let us consider the rays that reach a certain point $\mathrm{P}$ of the screen coming from the points of the arc AML. The difference $\ell$ of the distance $\mathrm{mP}$ from MP is equal to the length of the segment ms and is given by

$$
\ell=\frac{z^{2}}{2} \frac{a+b}{a b}
$$

where $z$ is the length of the arc Mm, $a$ is the distance $\mathrm{CA}$, and $b$ is the distance $\mathrm{AB}$. We are considering that

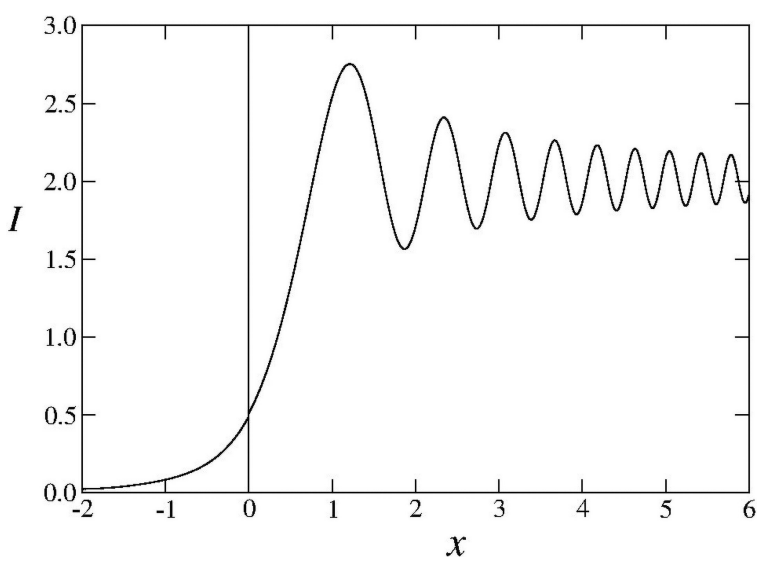

Figure 11: Intensity $I$ of diffraction fringes produced by a straight edge as a function of the distance $x$ to the limit of the geometric edge.

these distances are larger compared to $z$. To determine the wave at the point $\mathrm{P}$ we use the expressions 239 and 240 . The terms of these expressions are given by $d z \cos 2 \pi \ell / \lambda$ and $d z \sin 2 \pi \ell / \lambda$, respectively, where the waves have all the same intensity of vibrations and are proportional do $d z$. Integrating them we get

$$
B=\int d z \cos q z^{2}, \quad C=\int d z \sin q z^{2},
$$

where $q=\pi(a+r) / \operatorname{ar} \lambda$, and the integration is carried out from $z=-z_{0}$ to $z=\infty$, where $z_{0}$ is the distance BP. The intensity of vibrations is

$$
A=\sqrt{B^{2}+C^{2}} .
$$

The intensity of light at $\mathrm{P}$ is proportional to the square of the velocity and thus proportional to the square of the intensity of vibration, that is,

$$
A^{2}=B^{2}+C^{2}
$$

For convenience, let us define $x$ and $I$ by $x=z_{0} \sqrt{2 q / \pi}$ and $I=(2 q / \pi) A^{2}$. Then $I=\mathcal{B}^{2}+\mathcal{C}^{2}$, where

$$
\mathcal{B}=\int_{-x}^{\infty} d s \cos \frac{\pi}{2} s^{2}, \quad \mathcal{C}=\int_{-x}^{\infty} d s \sin \frac{\pi}{2} s^{2} .
$$

Fresnel carried out numerical integration of $\mathcal{B}$ and $\mathcal{C}$ from which he obtained $I$ and its maximum and minimum values for several values of $x$. We have also carried out the numerical integration and the results for $I$ are shown in Figure 11 .

\subsection{Polarization}

An object observed through a calcite crystal shows two images as a result of the double refraction of light. The double refraction in calcite was first described by Bartholin in 1669 and later by Huygens in his treatise on light published in 1690 . The two rays emerging from 


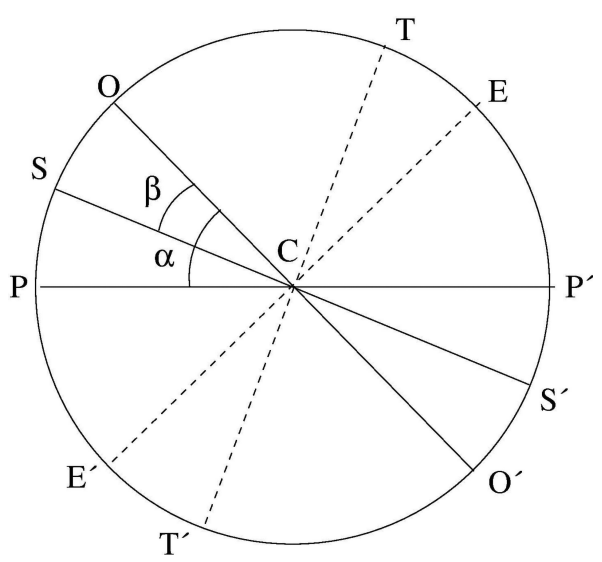

Figure 12: The incident light, polarized along the plane $\mathrm{PP}^{\prime}$, traverses a crystalline plate with principal section represented by $\mathrm{OO}^{\prime}$ and a rhomb of calcite with principal section $\mathrm{SS}^{\prime}$. The incident beam is divided into two beams polarized in the $\mathrm{CO}$ and CE directions.

a calcite are understood as polarized light. Another way of polarizing light, called polarization by reflection, was discovered by Malus in 1808. He observed through a calcite crystal that the sun light reflected on a glass window formed just one image instead of the expected two images, and assigned the effect to the polarization of the reflected light [4.

The polarization of light impelled the idea that a light ray might be composed by two distinct components that do not interfere one another. A crucial step in this direction was the observation by Fresnel and Arago that light polarized in orthogonal directions do not show the phenomena of diffraction, that is, they cannot interfere 89. According to Fresnel, Young was the first to propose, based on the optical properties of biaxial crystals, that the undulations of ether could be similar to the transversal waves of a stretched strings, and that the polarization of light was thus related to the orientation of the transverse vibration.

The explanation of Young, however, could not explain the existence of unpolarized light, and a more complete theory was carried out by Fresnel by postulating a pure transverse wave of light. This is contained in a paper on the tints developed in crystalline plates by polarization, published in 1821 [90, which we describe next.

Let us consider an incident light polarized along the plane $\mathrm{PP}^{\prime}$ which traverses the crystalline plate whose principal section $\mathrm{OO}^{\prime}$ makes an angle $\alpha$ with that plane, as seen in Figure 12. We ask for the intensity of the ordinary and extraordinary images determined by a homogeneous light of wavelength $\lambda$ considering that the principal section $\mathrm{SS}^{\prime}$ of the calcite makes an angle $\beta$ with $\mathrm{OO}^{\prime}$. If we denote by $F$ the intensity of the velocities of the ether molecules, which is the amplitude of the velocity oscillations, for the incident beam, then the intensity of light will be represented by $F^{2}$. After crossing the crystalline plate, the beam is divided into to other beams, which are orthogonally polarized in the directions $\mathrm{CO}$ and $\mathrm{CE}$. In accordance with the transverse wave postulate, the amplitudes of the velocities along $\mathrm{CO}$ and $\mathrm{CE}$ will follows the rules of decomposition of a vector parallel to PC. Thus the amplitude of the first or ordinary beam will be $F_{o}=F \cos \alpha$ and of the second or extraordinary beam will be $F_{e}=F \sin \alpha$. The intensity of light will be $F_{o}^{2}=F^{2} \cos ^{2} \alpha$ and $F_{e}^{2}=F^{2} \sin ^{2} \alpha$, in accordance with Malus's law.

Each of the beams is divided into two other beams by the action of the calcite crystal. Applying again the decomposition rule, the amplitudes of these four beams will be

$$
\begin{gathered}
F_{o o}=F \cos \alpha \cos \beta, \quad F_{o e}=F \cos \alpha \sin \beta, \\
F_{e o}=F \sin \alpha \sin \beta, \quad F_{e e}=-F \sin \alpha \cos \beta .
\end{gathered}
$$

Next, we have to find the intensity of the two wavetrains whose difference is o-e and corresponds to the amplitudes of oscillations $F_{o e}$ and $F_{e e}$. According to the rules given by equations 239, 240 and 242, the intensity of light is

$$
F_{o e}^{2}+F_{e e}^{2}+2 F_{o e} F_{e e} \cos 2 \pi \frac{\ell}{\lambda},
$$

where $\ell=o-e$, or

$$
\begin{aligned}
& F^{2}\left(\cos ^{2} \alpha \sin ^{2} \beta+\sin ^{2} \alpha \cos ^{2} \beta\right) \\
& \quad-2 F^{2} \cos \alpha \sin \beta \sin \alpha \cos \beta \cos 2 \pi \frac{\ell}{\lambda},
\end{aligned}
$$

which can be written in the simplified form

$$
F^{2} \sin ^{2}(\beta-\alpha)+F^{2} \sin 2 \beta \sin 2 \alpha \sin ^{2} \pi \frac{\ell}{\lambda} .
$$

An analogous calculation for the other two wavetrains give

$$
F^{2} \cos ^{2}(\beta-\alpha)+F^{2} \sin 2 \beta \sin 2 \alpha \sin ^{2} \pi \frac{\ell}{\lambda} .
$$

These two equations are the general formulas that give the intensity of each species of homogeneous light in the ordinary and extraordinary images in terms of the wavelengths $\lambda$ and the difference $c$ in their paths. As $\lambda$ is the same for both wavetrains, they corresponds to the same color but with distinct intensity, that is, with distinct tint. These two formulas, says Fresnel, can be compared with experimental observation by the aid of the empirical formula provided by Newton.

\section{Navier}

Claude Louis Navier was born in 1785 at Dijon, France. He entered the École Polytechnique in 1802 and the École de Ponts e Chaussées in 1804 from which he graduated in 1806. He lived for the rest of his life in the Saint-Germain-de-Prés quarter in Paris, where he 
died in 1836. From 1819, he taught the course in applied mechanics at the École des Ponts et Chaussées, becoming professor in 1830. In 1831, he also became professor at the École Polytechnique. During the period 1807-1820 he turned the mathematical analysis a fundamental tool of the civil engineer [91. He contributed to theory of elasticity and to the structural analysis but his major contribution is on fluid mechanics contained in a paper presented at the Academy of Sciences in 1822 and published in 1827 92.

In his paper on fluid mechanics [92, Navier restricted himself to the analysis of incompressible fluids. He represented a fluid by a collection of material points, or molecules, located at small distances from each other, and susceptible to change their position almost freely. The pressure inside the fluid tends to approach the molecules which reacts through repulsive forces. The forces of the molecules among themselves vary with the distance between molecules, being repulsive when the distance decreases and attractive when it increases. In addition to these forces, each molecule may also be subject to external forces such as gravity. We denote by $P, Q$, and $R$ the components of the external forces per unit volume.

The distinguish feature of the Navier approach lies on the consideration of the internal friction or viscosity, which is appropriate to describe viscous fluids. This is accomplished by postulating a second type of forces between two nearby molecules which emerges when their relative motion is nonzero. Navier assumes as a principle of the theory that these forces, which is the origin of the viscosity, are proportional to the difference in the velocities of the nearby molecules. This hypothesis had been introduced before by Newton in the Principles, section 6 of book 2 [33, 34]. For this reason, fluids following this rule, such as air and water, are called Newtonian fluids.

The fundamental principle used by Navier to find the equations of motion is that the sum of moments of the forces is equal to zero, which is the d'Alembert principle used by Lagrange, as we have seen above. Thus the problem is reduced to find the moments of the forces. Let us denote by $x, y$, and $z$ the rectangular coordinates of a molecule $\mathrm{M}$, and by $\delta x, \delta y$, and $\delta z$ the respective variations in the position. The sum of the moments of the external forces is

$$
\int(P \delta x+Q \delta y+R \delta z) d x d y d z
$$

The fundamental principle also includes the moment of the inertial force which is the mass multiplied by the acceleration. Denoting by $u, v$, and $w$ the velocity of $\mathrm{M}$ along the three rectangular direction, at the position $x$, $v$, and $z$, then the acceleration along the three directions are

$$
A=\frac{d u}{d t}+u \frac{d u}{d x}+v \frac{d u}{d y}+w \frac{d u}{d z}
$$

$$
\begin{aligned}
& B=\frac{d v}{d t}+u \frac{d v}{d x}+v \frac{d v}{d y}+w \frac{d v}{d z} \\
& C=\frac{d w}{d t}+u \frac{d w}{d x}+v \frac{d w}{d y}+w \frac{d w}{d z} .
\end{aligned}
$$

The sum of their moments is

$$
\int \rho(A \delta x+B \delta y+C \delta z) d x d y d z,
$$

where $\rho$ is the density, that is, the mass per unit volume. Next we determine the moments of the first type of forces between molecules alluded above.

Let us denote by $x, y$, and $z$ the coordinates of a certain molecule $\mathrm{M}$, as before, and by $x+\alpha, y+\beta$, and $z+\gamma$ the coordinates of a neighboring molecule $\mathrm{M}^{\prime}$. The distance between them is $r=\sqrt{\alpha^{2}+\beta^{2}+\gamma^{2}}$ and the force between them, denoted by $f(r)$, depends on $r$ as well as on the coordinates $x, y$, and $z$. Thus each molecule $\mathrm{M}$ is subject to forces of this type emanating from nearby molecules $\mathrm{M}^{\prime}$.

Considering that the fluid is the state of equilibrium one asks for the variation $\delta r$ in the distance $r$ that results from the variations $\delta x, \delta y$, and $\delta z$ in the position of the molecule $\mathrm{M}$. The answer is

$$
\begin{aligned}
r \delta r= & \frac{d \delta x}{d x} \alpha^{2}+\frac{d \delta y}{d y} \beta^{2}+\frac{d \delta z}{d z} \gamma^{2}+\frac{d \delta x}{d y} \alpha \beta+\frac{d \delta y}{d x} \alpha \beta \\
& +\frac{d \delta x}{d z} \alpha \gamma+\frac{d \delta z}{d x} \alpha \gamma+\frac{d \delta y}{d z} \beta \gamma+\frac{d \delta z}{d y} \beta \gamma .
\end{aligned}
$$

The moment of the force $f(r)$ is

$$
\iint f(r) \delta r d x d y d z d \alpha d \beta d \gamma
$$

Integrating in $\alpha, \beta$, and $\gamma$ by transforming these coordinates into spherical coordinates, we find

$$
\int p\left(\frac{d \delta x}{d x}+\frac{d \delta y}{d y}+\frac{d \delta z}{d z}\right) d x d y d z
$$

where

$$
p=\frac{4 \pi}{3} \iint_{0}^{\infty} r^{3} f(r) d r .
$$

An integration by parts of the integral (261), gives

$$
-\int\left(\frac{d p}{d x} \delta x+\frac{d p}{d y} \delta y+\frac{d p}{d z} \delta z\right) d x d y d z
$$

According to the fundamental principle, the sum of the moments given by 254, (258) with a minus sign, and (263) should vanish. As the variations $\delta x, \delta y$, and $\delta z$ are independent, their coefficients vanish and we find the equations of motion

$$
\begin{aligned}
& P-\frac{d p}{d x}-\rho A=0 \\
& Q-\frac{d p}{d y}-\rho B=0 \\
& R-\frac{d p}{d z}-\rho C=0 .
\end{aligned}
$$


These equations are the Euler equations (51), (52), and 53 .

Let us determine now the moments of the second type of forces, that are related to th internal friction. Denoting by $u, v$, and $w$ the components of the velocity of the molecule $\mathrm{M}$, as before, then the components of the velocity of the molecule $\mathrm{M}^{\prime}$ are $u+a, v+b$, and $w+c$, where

$$
\begin{aligned}
& a=\frac{d u}{d x} \alpha+\frac{d u}{d y} \beta+\frac{d u}{d z} \gamma \\
& b=\frac{d v}{d x} \alpha+\frac{d v}{d y} \beta+\frac{d v}{d z} \gamma \\
& c=\frac{d w}{d x} \alpha+\frac{d w}{d y} \beta+\frac{d w}{d z} \gamma
\end{aligned}
$$

and we are considering that $\alpha, \beta$, and $\gamma$ are small quantities. The difference of the velocities along the line joining the two molecules is

$$
V=\frac{\alpha}{r} a+\frac{\beta}{r} b+\frac{\gamma}{r} c .
$$

In accordance with the principle adopted by Navier, we understand that the force between the molecules $\mathrm{M}$ and $\mathrm{M}^{\prime}$ is proportional to increase in $V$ along the line joining the molecules and to a function $f(r)$ which decreases rapidly with the distance $r$ and vanishes when $r$ takes an appreciable value. Thus the components of this force along the $x, y$, and $z$ direction are $f(r)(d V / d x)$, $f(r)(d V / d y)$, and $f(r)(d V / d z)$, respectively. To determine the components $X, Y$, and $Z$ of the force should integrate in $\alpha, \beta$, and $\gamma$, that is,

$$
\begin{aligned}
X & =\int f(r) \frac{d V}{d x} d \alpha d \beta d \gamma \\
Y & =\int f(r) \frac{d V}{d y} d \alpha d \beta d \gamma \\
Z & =\int f(r) \frac{d V}{d z} d \alpha d \beta d \gamma
\end{aligned}
$$

The result of the integrals are

$$
\begin{aligned}
& X=\varepsilon\left(\frac{d^{2} u}{d x^{2}}+\frac{d^{2} u}{d y^{2}}+\frac{d^{2} u}{d z^{2}}\right), \\
& Y=\varepsilon\left(\frac{d^{2} v}{d x^{2}}+\frac{d^{2} v}{d y^{2}}+\frac{d^{2} v}{d z^{2}}\right), \\
& Z=\varepsilon\left(\frac{d^{2} w}{d x^{2}}+\frac{d^{2} w}{d y^{2}}+\frac{d^{2} w}{d z^{2}}\right),
\end{aligned}
$$

where

$$
\varepsilon=\frac{8 \pi}{30} \int_{0}^{\infty} d r r^{4} f(r) .
$$

Adding $X, Y$, and $Z$ to the equations (264), (265), and (266), we reach the Navier equations of motion,

$$
\begin{aligned}
& P-\frac{d p}{d x}-\rho A+X=0, \\
& Q-\frac{d p}{d y}-\rho B+Y=0, \\
& R-\frac{d p}{d z}-\rho C+Z=0,
\end{aligned}
$$

which in explicit form are

$$
\begin{array}{r}
\rho\left(\frac{d u}{d t}+u \frac{d u}{d x}+v \frac{d u}{d y}+w \frac{d u}{d z}\right) \\
=P-\frac{d p}{d x}+\varepsilon\left(\frac{d^{2} u}{d x^{2}}+\frac{d^{2} u}{d y^{2}}+\frac{d^{2} u}{d z^{2}}\right), \\
\rho\left(\frac{d v}{d t}+u \frac{d v}{d x}+v \frac{d v}{d y}+w \frac{d v}{d z}\right) \\
=Q-\frac{d p}{d y}+\varepsilon\left(\frac{d^{2} v}{d x^{2}}+\frac{d^{2} v}{d y^{2}}+\frac{d^{2} v}{d z^{2}}\right), \\
\rho\left(\frac{d w}{d t}+u \frac{d w}{d x}+v \frac{d w}{d y}+w \frac{d w}{d z}\right) \\
=R-\frac{d p}{d z}+\varepsilon\left(\frac{d^{2} w}{d x^{2}}+\frac{d^{2} w}{d y^{2}}+\frac{d^{2} w}{d z^{2}}\right) .
\end{array}
$$

We remark that $p$ is the pressure and $\varepsilon$ is the viscosity. These equations were also obtained by Stokes in 1845 [93], and are called Navier-Stokes equations [20].

\section{Conclusion}

We have examined the analytical physical theories that emerged after the introduction of the differential and integral calculus, restricting to the period from the very beginning of the eighteenth century up to the middle of the nineteenth century. We intend to analyze other analytical physical theories that appeared after this time in an other publication. The analytical theories that we have examined here employed the calculus, or analysis, as the underlying framework. This was a change in the theoretical approach as the previous theories from Euclid to Newton used basically the geometry methods. The mechanics of Lagrande was a typical and relevant example of these analytical theories. It is not by chance that the word analytic appears in the title of his treatise on mechanics.

The theories were examine on the light of the viewpoint stated above that a scientific theory consists of a framework composed by concepts and laws which are derived by a deductive reasoning from a set of fundamental laws and a set of primary concepts. In Table 1 we list the theories examined along with the main concepts, including the primary concepts. The primary, or primitive concepts, are undefinable concepts. In addition the framework is endowed with an interpretation which connects the abstract concepts to the real world. These correspondence are directly related to the 
Table 1: The author of a scientific theory is listed together with the abbreviated name of the main work where it is found, the year of its public presentation or publication, the subject of the theory, the main concepts including the primary concepts, and references.

\begin{tabular}{|c|c|c|c|c|c|}
\hline Author & Work & Year & Subject & Concepts & References \\
\hline Varignon & Motion in general & 1700 & particle dynamics & space, time, force & 1214 \\
\hline Euler & Mechanics & 1736 & particle dynamics & space, time, force & 23 \\
\hline D. Bernoulli & Hydrodynamics & 1738 & hydrodynamics & hydrodynamic pressure & $17+19$ \\
\hline Euler & Principles of fluid motion & 1757 & fluid mechanics & pressure, density & $26-28$ \\
\hline Euler, Lagrange & various papers & 1759 & sound propagation & pressure, density & 3641 \\
\hline Lagrange & Analytical Mechanics & 1788 & mechanics & space, time, mass, force & 31 \\
\hline Laplace & Celestial Mechanics & 1799 & mechanics & space, time, mass, force & 57 \\
\hline Laplace, Biot, Poisson & Theory of sound & 1808 & sound propagation & pressure, density & 6467 \\
\hline Poisson & Electricity distribution & 1812 & electricity & electric fluid & 73,74 \\
\hline Fresnel & Diffraction of light & 1819 & light diffraction & light wave & 87 \\
\hline Fresnel & Polarization of light & 1821 & light polarization & transverse light wave & 90 \\
\hline Fourier & Analytical Theory of Heat & 1822 & heat propagation & heat, temperature & 69 \\
\hline Poisson & Theory of magnetism & 1826 & magnetism & magnetic fluid & 76,77 \\
\hline Ampère & Electrodynamic phenomena & 1826 & electrodynamics & electric current & 79 \\
\hline Navier & Laws of fluid motion & 1827 & fluid mechanics & pressure, density, viscosity & 92 \\
\hline
\end{tabular}

measurements. We remark that not all concepts of a theory need to have a real counterpart, or, employing a terminology of quantum mechanics, need not be an observable.

\section{Acknowledgement}

I wish to acknowledge Tânia Tomé for the critical reading of the manuscript.

\section{References}

[1] M.J. de Oliveira, Rev. Bras. Ens. Fis. 43, e20200506 (2021).

[2] M.J. de Oliveira, Rev. Bras. Ens. Fis. 42, e20190192 (2020).

[3] R. Dugas, A History of Mechanics (Routledge and Kegan Paul, London, 1955).

[4] E. Segrè, From Fallling Bodies to Radio Waves (Freeman, New York, 1984).

[5] A.S.T. Pires, Evolução das Idéias da Fisica (Editora Livraria da Física, São Paulo, 2011), $2^{\text {a }}$ ed.

[6] L.R. Evangelista, Perspectivas em História da Física, Dos Babilônios à Sintese Newtoniana (Editora Ciência Moderna, São Paulo, 2011).

[7] L.R. Evangelista, Perspectivas em História da Física, Da Física dos Gases à Mecânica Estatística (Editora Livraria da Física, São Paulo, 2014).

[8] M.J. de Oliveira, Rev. Bras. Ens. Fis. 41, e20180307 (2019).

[9] T.L. Hankins, Jean d'Alembert, Science and the Enlightenment (Clarendon Press, Oxford, 1970).

[10] I. Grattan-Guinness (ed.), Landmark Writings in Western Mathematics, 1640-1940 (Elsevier, Amsterdam, 2005).

[11] C.C. Gillispie (ed.) Dictionary of Scientific Biography (Scribner, New York, 1976) v. 13, p. 584.

[12] P. Varignon, Mémoires de l'Académie Royale des Sciences, année 1700, p. 22 (1760).

[13] P. Varignon, Mémoires de l'Académie Royale des Sciences, année 1700, p. 83 (1760).
[14] P. Varignon, Mémoires de l'Académie Royale des Sciences, année 1700, p. 224 (1760).

[15] R. Dugas, Mechanics in the Seventeenth Century (Griffon, Neuchatel, 1958).

[16] C.C. Gillispie (ed.) Dictionary of Scientific Biography (Scribner, New York, 1970) v. 2, p. 36.

[17] D. Bernoulli, Hydrodynamica (Dulseckeri, Argentorati, 1738).

[18] D. Bernoulli and J. Bernoulli, Hydrodynamics and Hydraulics (Dover, New York, 1968).

[19] D. Bernoulli, Hydrodynamique (Blanchard, Paris, 2004).

[20] G.A. Tokaty, A History and Philosophy of Fluid Machanics (Dover, New York, 1971).

[21] C.C. Gillispie (ed.) Dictionary of Scientific Biography (Scribner, New York, 1971) v. 4, p. 467

[22] D. Suisky, Euler as Physicist (Springer, Berlin, 2019).

[23] L. Euler, Mechanica sive Motus Scientia Analytice Exposita (Academiae Scientiarum, Petropoli, 1736).

[24] A translation of 23] by I. Bruce is found in http://www. 17 cneturymaths.com

[25] D. Bernoulli, Commentarii Academiae Scientiarum Imperialis Petropolitanae 1, 126 (1728).

[26] L. Euler, Mémoires de l'académie des sciences de Berlin 11, 274 (1757).

[27] L. Euler, Mémoires de l'académie des sciences de Berlin 11, 316 (1757).

[28] L. Euler, Mémoires de l'académie des sciences de Berlin 11, 217 (1757)

[29] C.B. Boyer, The History of the Calculus and its Conceptual Development (Dover, New York, 1959).

[30] H. Lamb, Hydrodynamics (Cambridge University Press, Cambridge, 1895).

[31] J.L. Lagrange, Méchanique Analitique (Veuve Desaint, Paris, 1788).

[32] R.B. Lindsay (ed.), Acoustics: Historical and Philosophical Development (Dowden, Hutchinson and Ross, 1973).

[33] I. Newton, Philosophiae Naturalis Principia Mathematica (Streater, Londini, 1687).

[34] I. Newton, Mathematical Principles of Natural Philosophy (Motte, London, 1729).

[35] F. Cajori, Sir Isaac Newton's Mathematical Principles (University of California Press, Berkeley, 1974). 
[36] J.L. Lagrange, Miscellanea Taurinensia 1, 1 (1759).

[37] J. Lagrange, Miscellanea Taurinensia 2, 11 (1761).

[38] J. Lagrange, Miscellanea Taurinensia 2, 323 (1761).

[39] L. Euler, Mémoires de l'académie des sciences de Berlin 15, 185 (1766).

[40] L. Euler, Mémoires de l'académie des sciences de Berlin 15, 210 (1766).

[41] L. Euler, Mémoires de l'académie des sciences de Berlin 15, 241 (1766).

[42] A.D. Pierce, Acoustics (Springer, Cham, 2019) 3rd. ed.

[43] J. d'Alembert, Mémoires de l'Académie des Sciences de Berlin, année 1747, 214 (1749).

[44] B. Taylor, Philosophical Transactions of the Royal Society 28, 26 (1714).

[45] C.C. Gillispie (ed.) Dictionary of Scientific Biography (Scribner, New York, 1973) v. 7, p. 559.

[46] L. Euler, Methodus inviniendi lineas curvas maximi minimive proprietate gaudentes (Bousquet, Lausannae et Genevae, 1744).

[47] J.L. Lagrange, Miscellanea Taurinensia 1, 18 (1759).

[48] J.L. Lagrange, Miscellanea Taurinensia 2, 175 (1760).

[49] J.L. Lagrange, Analytical Mechanics (Springer, Dordrecht, 1997).

[50] J.L. Lagrange, Miscellanea Taurinensia 2, 195 (1760).

[51] J. d'Alembert Traité de Dynamique (David l'aîne, Paris, 1743).

[52] L. Euler, Novi Commentarii Academiae Scientiarum Imperialis Petropolitanae 10, 51 (1766).

[53] L. Euler, Novi Commentarii Academiae Scientiarum Imperialis Petropolitanae 10, 94 (1766).

[54] W.R. Hamilton, Philosophical Transactions of the Royal Society of London 124, 247 (1834).

[55] W.R. Hamilton, Philosophical Transactions of the Royal Society of London 125, 95 (1835).

[56] C.C. Gillispie (ed.) Dictionary of Scientific Biography (Scribner, New York, 1981) v. 15, p. 273.

[57] P.S. Laplace, Traité de Mécanique Céleste (Duprat, Paris, 1799), tome premier.

[58] P.S. Laplace, Traité de Mécanique Céleste (Duprat, Paris, 1799), tome second.

[59] P.S. Laplace, Traité de Mécanique Céleste (Duprat, Paris, 1802), tome troisième.

[60] P.S. Laplace, Traité de Mécanique Céleste (Duprat, Paris, 1805), tome quatrième.

[61] P.S. Laplace, Traité de Mécanique Céleste (Bachelier, Paris, 1825), tome cinquième.

[62] P.S. Laplace, Mémoires de Mathématique et de Physique de l'Académie Royale des Sciences, année 1782, p. 113 (1785).

[63] B.S. Finn, Isis 55, 7 (1964).

[64] P.S. Laplace, Annales de Chimie et de Physique 3, 238 (1816).

[65] P.S. Laplace, Connaissance des Tems ou des Mouvemens Célestes à l'Usage des Astronomes et des Navigateurs pour l'an 1825 1, 302 (1822).

[66] J.B. Biot, Journal de Physique, de Chimie, d'Histoire Naturelle et des Arts 55, 173 (1802).

[67] S.D. Poisson, Journal de l'École Polytechnique 7, 319 (1808).
[68] J. Herivel, Joseph Fourier, the Man and the Physicist (Clarendon Press, Oxford, 1975).

[69] J. Fourier, Théorie Analytique de la Chaleur (Firmin Didot, Paris, 1822).

[70] J. Fourier, Analytical Theory of Heat (Cambridge University Press, Cambridge, 1878).

[71] S.G. Brush, The Kind of Motion we Call Heat (North Holland, Amsterdam, 1986) v. 2.

[72] C.C. Gillispie (ed.) Dictionary of Scientific Biography (Scribner, New York, 1978) v. 15, p. 480.

[73] S.D. Poisson, Mémoires de la Classe de Sciences Mathématiques et Physiques de l'Institut Impérial de France, première partie année 1811, premiére partie, p. 1 (1812).

[74] S.D. Poisson, Mémoires de la Classe de Sciences Mathématiques et Physiques de l'Institut Impérial de France, seconde partie année 1811, premiére partie, p. 183 (1812).

[75] S.D. Poisson, Bulletin des Sciences de la Societé Philomatique 3, 388 (1813).

[76] S.D. Poisson, Mémoires de l'Académie Royale de Sciences Mathématiques et Physiques de l'Institut de France annees 1821 et 1822, 5, 247 (1826).

[77] S.D. Poisson, Mémoires de l'Académie Royale de Sciences Mathématiques et Physiques de l'Institut de France annees 1821 et 1822, 5, 448 (1826).

[78] J.R. Hofmann, André-Marie Ampère (Cambridge University Press, Cambridge, 1995).

[79] A.M. Ampère, Théorie des Phénomènes ÉlectroDynamiques (Méquignon-Marvis, Paris, 1826).

[80] R.A.R. Tricker, Early Electrodynamics: The First Law of Circulation (Pergamon Press, Oxford, 1965).

[81] F. Savary, Journal de Physique, de Chimie et d'Histoire Naturelle 96, 1 (1823).

[82] J. B. Biot et F. Savart, Annales de Chimie et de Physique 15, $222(1820)$

[83] J.B. Biot, Journal de Savans, avril 1821, p. 221 (1821).

[84] J.B. Biot, Précis Élémentaire de Physique Expérimentale (Deterville, Paris, 1821), tome 2, second édition.

[85] J.B. Biot, Précis Élémentaire de Physique Expérimentale (Deterville, Paris, 1824) tome 2, troisième édition.

[86] C.C. Gillispie (ed.) Dictionary of Scientific Biography (Scribner, New York, 1972) v. 5, p. 165.

[87] A. Fresnel, Mémoires de l'Académie Royale des Sciences de l'Institut de France, années 1820 et 1821, 5, 339 (1826).

[88] A. Fresnel, Annales de Chimie et de Physique 1, 239 (1816).

[89] A. Fresnel and F. Arago, Annales de Chimie et de Physique 10, 288 (1819).

[90] A. Fresnel, Annales de Chimie et de Physique 17, 102 (1821).

[91] C.C. Gillispie (ed.) Dictionary of Scientific Biography (Scribner, New York, 1974) v. 10, p. 2.

[92] C.L. Navier, Mémoires de l'Académie Royale des Sciences de l'Institut de France, année 1823, 6, 389 (1827).

[93] G. Stokes, Transactions of the Cambridge Philosophical Society 8, 287 (1849). 